\title{
Demographic and Geographic Patterns of Cetacean-based Food Product Consumption and Potential Mercury Exposure within a Caribbean Whaling Community
}

\author{
Russell Fielding ( $\square$ rfielding@coastal.edu ) \\ https://orcid.org/0000-0002-5401-2141 \\ Jeremy J Kiszka \\ Florida International University https://orcid.org/0000-0003-1095-8979 \\ Catherine Macdonald \\ Meaghan A. McCormack \\ Texas State University \\ Jessica Dutton \\ Texas State University \\ Alison D. Ollivierre \\ Tombolo Maps \& Design \\ Jay Arnett \\ University of Colorado Denver - Anschutz Medical Campus \\ Molly Elkins \\ University of the South \\ Niko A. Darby \\ University of the South \\ Hannah-Marie Garcia \\ University of the South/University of Delaware \\ Suzanne Skinner \\ University of the South \\ Haley Tucker \\ University of the South \\ Vincent Reid \\ Barrouallie Whalers Project
}

Field School Scientific Training/University of Miami Rosenstiel School of Marine and Atmospheric Science

\section{Research}

Keywords: cetaceans, contaminant exposure, Caribbean, diet, ecotoxicology, mercury, methylmercury, provisional tolerable weekly intake, survey, whaling

Posted Date: June 9th, 2020

DOI: https://doi.org/10.21203/rs.3.rs-33554/v1

License: (c) (i) This work is licensed under a Creative Commons Attribution 4.0 International License. Read Full License 
Version of Record: A version of this preprint was published at Human and Ecological Risk Assessment: An International Journal on January 13th, 2021. See the published version at https://doi.org/10.1080/10807039.2020.1870865. 


\section{Abstract}

Background: Exposure to mercury $(\mathrm{Hg})$ through the consumption of seafood is a major public health concern. The tissues of high trophic level marine organisms, such as cetaceans, often contain high concentrations of $\mathrm{Hg}$ compared to lower trophic level species. The archipelagic Caribbean nation of St. Vincent \& the Grenadines supports two related whaling operations, which produce food from cetaceans for human consumption. A recent study reported that $\mathrm{Hg}$ concentrations in tissue samples from one of these whaling operations exceed recommended consumption limits. Our objective was to determine the role of cetacean-based food products in the diet of the Vincentian population as a proxy for exposure to $\mathrm{MeHg}$.

Methods: We conducted interviews with 921 members of the Vincentian public. We asked questions about the types of food consumed, frequency of consumption, and quantities consumed. Based upon provisional tolerable weekly intake (PTWI) guidelines for methylmercury (MeHg) from the Food and Agricultural Organization (FAO) and the World Health Organization (WHO), we determined safe weekly consumption amounts for specific cetacean-based food products, based upon reported consumption patterns.

Results: We found cetacean-based food products to be included in the diet of 77.4 percent of respondents. Respondents' gender and geographical home region are the most predictive attributes for cetacean-based food product consumption, whereas socioeconomic status and age play a minor role and no role, respectively. Frequency and amount of consumption vary, but generally exceed calculated safe weekly consumption amounts.

Conclusions: The consumption of cetacean-based food products by members of the Vincentian public may represent a public health risk, as data indicate that a large portion of the population may be exposed to concentrations of MeHg in excess of recommended limits. Any potential action to address this risk is likely to be most effective if targeted toward the specific high-consumption population segments identified by our study.

\section{Background}

Mercury $(\mathrm{Hg})$ contamination in seafood is a major public health concern, and risk assessments are critical to efforts to manage rates of exposure (Kojadinovic et al. 2006; Grandjean 2013; Olmedo et al. 2013). Due to the biomagnification of methylmercury (MeHg; the most toxic and abundant form of $\mathrm{Hg}$ in seafood) in marine food webs, high trophic-level marine organisms with long lifespans-including tunas, billfish, sharks, and cetaceans-have the potential to contain high $\mathrm{Hg}$ levels (Wagemann et al. 1997; Kehrig et al. 2002; Kojadinovic et al. 2006). Cetacean-based food products are consumed globally at varying levels, and their consumption has been increasing in several regions around the world during recent years (Clapham and Van Waerebeek 2007; Robards and Reeves 2011). In some cases, this increase is in response to food shortages exacerbated by climate change and other human impacts (Alter et al. 2010). Studies have confirmed the increasing risk related to the consumption of cetacean-based food products due to the presence of high contaminant levels, particularly $\mathrm{Hg}$ (e.g. Simmonds et al. 2002; Endo and Haraguchi 2010).

The archipelagic Caribbean nation of St. Vincent \& the Grenadines (SVG; Fig. 1) supports two distinct, yet related, whaling operations, both of which produce food for human consumption. The first, based on the island of Bequia, holds an International Whaling Commission (IWC)-sanctioned aboriginal subsistence whaling quota to take up to four humpback whales (Megaptera novaeangliae) per year (Adams 1971; Ward 1995). The second, based in the town of Barrouallie on the main island of St. Vincent, takes short-finned pilot whales (Globicephala macrorhynchus) and other small cetaceans not managed by the IWC (Gillespie 2001). These include Atlantic spotted dolphins (Stenella frontalis), false killer whales (Pseudorca crassidens), Fraser's dolphins (Lagenodelphis hosei), killer whales (Orcinus orca), Risso's dolphins (Grampus griseus), and spinner dolphins (Stenella longirostris) (Adams 1973; Caldwell and Caldwell 1975; Fielding 2018). The two whaling operations in SVG produce meat that is consumed fresh, dried (often then rehydrated), or "doved" (cooked and stored in its own oil); blubber, which is consumed after having been "rendered" or heated until it expresses-and is then fried in-its oil; and oil itself, which is bottled and used as a health tonic and treatment for colds. 
[Figure 1 here]

Whaling was introduced to the Eastern Caribbean through interaction with the New England-based "Yankee Whaler" fleet during the 19th century (Romero and Cresswell 2005). Both the humpback whale-focused operation on Bequia and the operation targeting small cetaceans from the main island of St. Vincent grew out of this introduction. Historically, food produced from small cetaceans was a last resort for the poor-considered relatively unpalatable-and was one of the least expensive local protein options (Adams 1980). Today, the consumption of cetacean meat has also become a cultural signifier, or an expression of Vincentian identity (Zane 1999; Fielding 2018).

Food products derived from small cetaceans are distributed through formal networks within the main island of St. Vincent. This consists mainly of established vendors selling dried meat or cooked blubber from stalls at the island's major fish markets and mobile vendors traveling throughout the island and setting up temporary stalls or tables on street corners from which to sell small cetacean products, but other methods of acquisition exist, particularly for food products derived from internal organs such as liver and kidney (Fielding 2014, 2018). The Grenadines rely more upon informal distribution networks (e.g. a family member bringing products from St. Vincent) for small cetacean products, although during periods of increased supply, St. Vincent-based vendors will occasionally travel by ferry throughout the Grenadines. Food products derived from humpback whales are distributed immediately following the catch of a whale-which occurs a maximum of four times per year. The primary distribution occurs in Bequia, but humpback whale products are distributed secondarily, mainly through informal family-based networks, throughout St. Vincent and the rest of the Grenadines. The prices of cetacean-based food products remain accessible: EC $\$ 2.50$ (approx. US\$1.00) per bundle for meat from small cetaceans and EC\$5.00 (approx. US \$2.00) per pound for meat from humpback whales (IWC 2015).

As long-lived and high trophic-level marine predators, the small cetaceans taken in the Barrouallie operation are subject to high concentrations of $\mathrm{Hg}$. Recent analysis by McCormack et al. (2020) suggests that mean concentrations of total mercury ( $\mathrm{THg}$ ) in muscle, blubber, and internal organs (liver and kidney) from five species taken in Barrouallie (Table 1) far exceed the advisory level for human consumption of $1.0 \mu \mathrm{g} / \mathrm{g}$ wet-weight determined by the United Nations Food \& Agriculture Organization and the World Health Organization (FAO/WHO 2011). Tissue samples from humpback whales caught in Bequia have not been analyzed for contaminant levels, concentrations of which are known to vary widely by region in this species (Wise et al. 2018, 2019a, 2019b). 
Table 1

Mean, minimum, and maximum THg wet-weight concentrations in SVG cetacean tissue. Summarized from McCormack et al. (2020).

\begin{tabular}{|c|c|c|c|c|}
\hline Species & $\begin{array}{l}\text { Tissue } \\
\text { Type (n) }\end{array}$ & $\begin{array}{l}\text { Mean THg ww concentration } \\
(\mu \mathrm{g} / \mathrm{g}) \pm \text { std. dev. }\end{array}$ & $\begin{array}{l}\text { Minimum THg ww } \\
\text { concentration }(\mu \mathrm{g} / \mathrm{g})\end{array}$ & $\begin{array}{l}\text { Maximum THg ww } \\
\text { concentration }(\mu \mathrm{g} / \mathrm{g})\end{array}$ \\
\hline \multirow{2}{*}{$\begin{array}{l}\text { False killer whale } \\
\text { (Pseudorca } \\
\text { crassidens) }\end{array}$} & $\begin{array}{l}\text { muscle } \\
(21)\end{array}$ & $4.35 \pm 5.72$ & 0.684 & 26.0 \\
\hline & $\begin{array}{l}\text { blubber } \\
\text { (5) }\end{array}$ & $2.72 \pm 1.79$ & 1.57 & 5.89 \\
\hline \multirow[t]{2}{*}{$\begin{array}{l}\text { Killer whale } \\
\text { (Orcinus orca) }\end{array}$} & $\begin{array}{l}\text { muscle } \\
(12)\end{array}$ & $48.4 \pm 71.0$ & 6.50 & 202 \\
\hline & $\begin{array}{l}\text { blubber } \\
\text { (6) }\end{array}$ & $5.04 \pm 2.55$ & 1.13 & 7.63 \\
\hline \multirow[t]{2}{*}{$\begin{array}{l}\text { Risso's dolphin } \\
\text { (Grampus griseus) }\end{array}$} & $\begin{array}{l}\text { muscle } \\
(3)\end{array}$ & $11.0 \pm 15.1$ & 1.12 & 28.4 \\
\hline & $\begin{array}{l}\text { blubber } \\
\text { (1) }\end{array}$ & $0.270 \pm N D$ & ND & ND \\
\hline \multirow{2}{*}{$\begin{array}{l}\text { Short-finned pilot } \\
\text { whale } \\
\text { (Globicephala } \\
\text { macrorhynchus) }\end{array}$} & $\begin{array}{l}\text { muscle } \\
(21)\end{array}$ & $20.5 \pm 45.8$ & 0.906 & 186 \\
\hline & $\begin{array}{l}\text { blubber } \\
(23)\end{array}$ & $1.35 \pm 0.671$ & 0.125 & 3.24 \\
\hline \multirow[t]{2}{*}{$\overline{\text { (Stenella spp.) }}$} & $\begin{array}{l}\text { muscle } \\
(2)\end{array}$ & $3.22 \pm N D$ & 2.09 & 4.34 \\
\hline & $\begin{array}{l}\text { blubber } \\
\text { (1) }\end{array}$ & $3.79 \pm N D$ & ND & ND \\
\hline
\end{tabular}

The use of cetaceans as a local food source in SVG potentially exposes human consumers to high concentrations of $\mathrm{Hg}$ (Fielding 2018; McCormack et al. 2020). The degree to which individuals are exposed to $\mathrm{Hg}$ is based, in part, upon individual rates of consumption of cetacean-based food products. In order to determine the demographic and geographic patterns to the consumption of cetacean-based food products in SVG, we undertook an interview-based survey of local dietary habits across the major, inhabited islands of the archipelago. While previous seafood-related dietary surveys have been conducted in SVG (e.g. Adams 1980; Josupeit 2011), our study is the first to focus explicitly on the consumption of cetacean-based food products and the first designed specifically to measure and map the potential exposure to public health risks presented by environmental contaminants concentrated therein.

\section{Methods}

\section{Data Collection}

During July and August 2018, we conducted in-person individual interviews with 921 adults ( $\geq 18$ years) living in SVG. This sample constitutes $1.3 \%$ of the country's adult population, according to the most recent census estimates (Ministry of Finance 2019). Our interviews were conducted on four of the country's eight inhabited islands: St. Vincent, Bequia, Mayreau, and Union Island. More important to determining the geographic patterns of consumption than the interview locations, however, were the hometowns that our respondents reported; we interviewed individuals claiming 69 hometowns throughout SVG, which we then grouped into geographical regions. The sparsely inhabited interior of the main island (Fielding \& Ollivierre 2017) was not included in our survey. 
Participants were recruited through various methods including encounters in public spaces, personal introductions through our local contacts, and a snowball sampling method (Coleman 1958-59).

Our sample also represents the country's demographics reasonably well (Table 2). We distinguished ages at the upper end of the range differently than the census (80-89 and 90-99 in our survey, as opposed to 80-84 and 85+ in the census). For comparison purposes, we have collapsed both ranges into the 80 + category in Table 2. Our age-specific analysis does not include data from respondents for whom we have no age information, nor from respondents in the oldest age category (9099 years), owing to the low sample size $(n=2)$ in that range.

Table 2

Demographic data from the 2012 SVG Census, compared this study's sample.

\begin{tabular}{|c|c|c|c|}
\hline Category & Criteria & Census percentage & Survey percentage \\
\hline \multirow[t]{2}{*}{ Gender } & Female & $49.1 \%$ & $39.5 \%$ \\
\hline & Male & $50.1 \%$ & $60.5 \%$ \\
\hline \multirow[t]{8}{*}{ Age range } & $18-29$ & $27.4 \%$ & $17.6 \%$ \\
\hline & $30-39$ & $20.2 \%$ & $17.5 \%$ \\
\hline & $40-49$ & $19.2 \%$ & $21.2 \%$ \\
\hline & $50-59$ & $15.2 \%$ & $17.5 \%$ \\
\hline & $60-69$ & $8.7 \%$ & $14.5 \%$ \\
\hline & $70-79$ & $5.9 \%$ & $8.0 \%$ \\
\hline & $80+$ & $3.5 \%$ & $2.8 \%$ \\
\hline & No Data & $0.0 \%$ & $0.7 \%$ \\
\hline \multirow[t]{14}{*}{ Occupation } & Managers & $4 \%$ & $6.8 \%$ \\
\hline & Professionals & $11 \%$ & $4.6 \%$ \\
\hline & Technicians and associate professionals & $7 \%$ & $1.3 \%$ \\
\hline & Clerical support workers & $6 \%$ & $1.5 \%$ \\
\hline & Service and sales workers & $26 \%$ & $11.0 \%$ \\
\hline & Skilled agricultural, forestry and fishery workers & $13 \%$ & $15.0 \%$ \\
\hline & Craft and related trades workers & $13 \%$ & $7.4 \%$ \\
\hline & Plant and machine operators, and assemblers & $5 \%$ & $5.5 \%$ \\
\hline & Elementary occupations & $13 \%$ & $6.0 \%$ \\
\hline & Military & - & $0.2 \%$ \\
\hline & Students & - & $0.8 \%$ \\
\hline & Retired & - & $5.6 \%$ \\
\hline & Unemployed & - & $5.6 \%$ \\
\hline & Not Stated & $1 \%$ & $33 \%$ \\
\hline
\end{tabular}

As for occupational categories, we based our distinctions upon the 2008 International Standard Classification of Occupations (ISOC-08) published by the United Nations' International Labour Office (ILO) with some modifications. Owing to 
the presence of unemployed, retired respondents, and students, we added these categories to the list. Respondents who reported being retired from a specific occupation were categorized in that occupation. Those who simply reported being retired and did not elaborate were categorized only as "retired." A 2019 International Monetary Fund report found the unemployment rate in SVG to be greater than 20\% (James et al. 2019). In our sample, $5.6 \%$ of respondents reported being unemployed, but a much larger subset (264 individuals, i.e. $28.7 \%$ of the sample) chose not to state their occupation.

[Table 2 here]

Food products derived from a variety of small cetaceans in the Barrouallie operation are all sold under the local name, "blackfish," without reference to the species of origin (Fielding 2014). Consumers are almost never aware of which species they are consuming. As such, we did not distinguish beyond this designation in our interviews; rather, we used the term "blackfish" to refer to the entire suite of small cetaceans taken in the Barrouallie operation, and "whale" to refer only to the humpback whales taken in Bequia, again following local usage of the term. Cooked blubber was referred to using the local term, "crisps" (pronounced with the first "s" silent, as crips). When respondents referred to other food products with unfamiliar local terms, we transcribed the terms phonetically and consulted with our local collaborators to determine the precise food product(s) being discussed.

Interviews were semi-structured in format and conversational in tone. They were conducted by teams, each consisting of two US-based undergraduate students and one local collaborator. Each interview included key questions about the respondent's identity and regular diet. Within this framework, we obtained demographic and dietary data, including details such as whether the respondent consumes food products derived from cetaceans at all (distinguishing between the small cetaceans taken in Barrouallie and the humpback whales taken in Bequia), which products the respondent consumes (meat, blubber, and oil), quantity of consumption per unit of time, and reasons why cetacean-based food products are not consumed (by respondents who report not consuming them). During each interview, interviewers took handwritten notes, which we transcribed into a Microsoft Excel spreadsheet at the end of each day as part of an iterative debriefing process which allowed continuous refinement of questions in response to results.

\section{Data analysis}

Data with identifiable personal information removed were analyzed with six primary variables collected for each of the 921 individuals included in the final study. Consumption of food products derived from small cetaceans and from humpback whales were each analyzed separately as primary outcomes. Respondents' age category, gender, home region, and occupational category were included as primary explanatory variables. Likelihood ratio tests were conducted to determine the overall significance of each factor and Akaike information criteria (AIC) values were used to compare model fit and guide final model selection. Given the binary outcome under consideration-whether a person does or does not consume food products derived from small cetaceans or humpback whales-logistic regression was used to generate estimated adjusted odds ratios and $95 \%$ confidence intervals for the increased or decreased odds of consumption based on the explanatory variables included in the best-fitting model. P-values of $<0.05$ were considered statistically significant. Statistical analyses were conducted in $R$ with models estimated as generalized linear models with the $\mathrm{g} / \mathrm{m}()$ function and a logit link function ( $R$ Core Team 2016).

\section{Determination of safe weekly consumption amounts}

To minimize negative health effects resulting from the ingestion of contaminated food products, the FAO/WHO provides estimates of the provisional tolerable weekly intake (PTWI). The PTWI equates to the amount of a contaminant per unit of body weight that can be ingested weekly without adverse health effects. For MeHg, the PTWI is currently $1.6 \mu \mathrm{g}$ per kilogram of body weight (JECFA 2006). McCormack et al. (2020), who first presented the current THg concentrations for small cetaceans in SVG, estimated the amount of muscle, blubber, liver, and kidney that could be consumed weekly by a $60 \mathrm{~kg}$ person so as to not exceed the PTWI. That study estimated these values by taking the mean wet weight THg concentration for all species combined for each tissue type and determining the amount of MeHg present in each tissue based on one 
tissue-specific MeHg percentage taken from the literature. While this resulted in a coarse estimation, it did not account for variation among species; however, because consumers in St. Vincent are not aware of the species they are consuming, the coarse estimation was appropriate given the scope of that study.

To account for variability in THg concentrations and the percentage MeHg within and among species, in the present study, we estimated the amount of each tissue type that could be consumed weekly without exceeding the PTWI using a more detailed approach. Using the data from McCormack et al. (2020), for each tissue sample, the amount of MeHg was estimated by species, based upon a range of tissue-specific MeHg percentages taken from the literature (Gaskin et al. 1974; Arima and Nagakura 1979; Itano et al. 1984; Palmisano et al. 1995; Storelli et al. 1998, 1999; Cardellicchio et al. 2002; Chen et al. 2002; Bustamante et al. 2003; JMHLW 2003; Ruelas-Inzunza et al. 2003; Endo et al. 2005, 2006; Capelli et al. 2008; Fielding and Evans 2014; Sakamoto et al. 2015). If values were not found for a specific tissue, the mean percent MeHg of other species was substituted. The maximum amount of $\mathrm{MeHg}(\mathrm{in} \mu \mathrm{g}$ ) that a $60 \mathrm{~kg}$ person can ingest each week without exceeding the PTWI $(96 \mu \mathrm{g})$ was then divided by the concentration of $\mathrm{MeHg}$ in each sample to determine the amount of tissue that can safely be consumed per week.

\section{Results}

Cetacean-based food products are highly popular among consumers in SVG, with $77.4 \%$ of all respondents indicating that they do consume at least one of the following products: small cetacean meat, small cetacean blubber, small cetacean oil, humpback whale meat, humpback whale blubber, or humpback whale oil. The popularity of cetacean-based food products varies, however, across demographic categories and geographic areas. (Table 3, Table 4). When determining which characteristics contribute to increased consumption of food products derived from small cetaceans, gender $\left(\chi^{2}=15.21, p<\right.$ $0.001, \mathrm{df}=1)$ and home region $\left(\chi^{2}(13, n=921)=94.99, \mathrm{p}<0.001\right)$ proved to be highly significant predictors. Occupational category $\left(\chi^{2}(9, n=921)=16.71, p=0.054\right)$ was determined to be an important precision variable and included in the final model even though not significant at the $p<0.05$ level. Age $\left(\chi^{2}(8, n=921)=5.79, p=0.67\right)$ proved not to be predictive and therefore not included in our final model. For the consumption of food products derived from humpback whales, home region $\left(\chi^{2}(13, n=921)=219.82, p<0.001\right)$ is the primary predictor with gender $\left(\chi^{2}(1, n=921)=9.00, p=0.003\right)$ also highly significant. Occupational category $\left(\chi^{2}(9, n=921)=16.04, p=0.066\right)$ was marginally significant whereas age $\left(\chi^{2}(8, n=921)\right.$ $=5.83, p=0.67$ ) again was not. Within these categories, variation in likelihood of consumption exists both directionally (higher versus lower odds of consumption) and in magnitude (how much the odds of consumption change). 
Table 3

The portion of respondents, by demographic and geographic categories, confirming consumption of cetacean-based food products.

\begin{tabular}{|c|c|c|c|c|c|c|c|c|c|c|}
\hline \multirow[t]{2}{*}{ Category } & \multirow[t]{2}{*}{ Criteria } & \multicolumn{4}{|c|}{ Small cetacean products } & \multicolumn{4}{|c|}{ Humpback whale products } & \multirow{2}{*}{$\begin{array}{l}\text { Any } \\
\text { cetacean- } \\
\text { based } \\
\text { food } \\
\text { product }\end{array}$} \\
\hline & & Meat & Blubber & Oil & Any & Meat & Blubber & Oil & Any & \\
\hline $\begin{array}{l}\text { Entire } \\
\text { sample }\end{array}$ & & $59.7 \%$ & $37.7 \%$ & $29.4 \%$ & $66.0 \%$ & $30.3 \%$ & $17.7 \%$ & $22.0 \%$ & $36.0 \%$ & $77.4 \%$ \\
\hline \multirow[t]{2}{*}{ Gender } & male & $64.3 \%$ & $41.7 \%$ & $34.5 \%$ & $71.6 \%$ & $33.4 \%$ & $20.1 \%$ & $23.9 \%$ & $39.5 \%$ & $82.8 \%$ \\
\hline & female & $52.7 \%$ & $31.6 \%$ & $21.7 \%$ & $57.4 \%$ & $25.5 \%$ & $14.0 \%$ & $19.2 \%$ & $30.8 \%$ & $69.2 \%$ \\
\hline \multirow[t]{9}{*}{ Age range } & $18-29$ & $54.3 \%$ & $30.9 \%$ & $22.8 \%$ & $63.0 \%$ & $29.6 \%$ & $17.9 \%$ & $24.1 \%$ & $37.0 \%$ & $75.3 \%$ \\
\hline & $30-39$ & $62.1 \%$ & $37.9 \%$ & $32.9 \%$ & $70.2 \%$ & $28.6 \%$ & $12.4 \%$ & $20.5 \%$ & $36.6 \%$ & $80.7 \%$ \\
\hline & $40-49$ & $65.3 \%$ & $43.4 \%$ & $29.6 \%$ & $71.4 \%$ & $28.6 \%$ & $15.3 \%$ & $19.9 \%$ & $34.2 \%$ & $80.1 \%$ \\
\hline & $50-59$ & $58.4 \%$ & $37.3 \%$ & $35.4 \%$ & $64.0 \%$ & $32.9 \%$ & $22.4 \%$ & $22.4 \%$ & $39.8 \%$ & $78.3 \%$ \\
\hline & $60-69$ & $54.8 \%$ & $40.7 \%$ & $26.7 \%$ & $60.7 \%$ & $34.1 \%$ & $22.2 \%$ & $27.4 \%$ & $37.0 \%$ & $75.6 \%$ \\
\hline & $70-79$ & $62.2 \%$ & $31.1 \%$ & $32.4 \%$ & $63.5 \%$ & $25.7 \%$ & $16.2 \%$ & $18.9 \%$ & $28.4 \%$ & $73.0 \%$ \\
\hline & $80-89$ & $61.5 \%$ & $42.3 \%$ & $15.4 \%$ & $65.4 \%$ & $38.5 \%$ & $19.2 \%$ & $15.4 \%$ & $38.5 \%$ & $69.2 \%$ \\
\hline & $90-99$ & $50.0 \%$ & $50.0 \%$ & $50.0 \%$ & $50.0 \%$ & $0.0 \%$ & $0.0 \%$ & $0.0 \%$ & $0.0 \%$ & $50.0 \%$ \\
\hline & ND & $75.0 \%$ & $25.0 \%$ & $25.0 \%$ & $75.0 \%$ & $25.0 \%$ & $25.0 \%$ & $25.0 \%$ & $25.0 \%$ & $100 \%$ \\
\hline \multirow[t]{11}{*}{ Occupation } & unemployed & $61.5 \%$ & $32.7 \%$ & $25.0 \%$ & $69.2 \%$ & $36.5 \%$ & $21.2 \%$ & $21.2 \%$ & $38.5 \%$ & $80.8 \%$ \\
\hline & Managers & $57.1 \%$ & $41.3 \%$ & $34.9 \%$ & $66.7 \%$ & $41.3 \%$ & $22.2 \%$ & $20.6 \%$ & $44.4 \%$ & $74.6 \%$ \\
\hline & Professionals & $54.8 \%$ & $28.6 \%$ & $21.4 \%$ & $52.4 \%$ & $47.6 \%$ & $35.7 \%$ & $33.3 \%$ & $45.2 \%$ & $69.0 \%$ \\
\hline & Technicians & $50.0 \%$ & $33.3 \%$ & $25.0 \%$ & $50.0 \%$ & $16.7 \%$ & $8.3 \%$ & $25.0 \%$ & $25.0 \%$ & $58.3 \%$ \\
\hline & $\begin{array}{l}\text { Clerical } \\
\text { workers }\end{array}$ & $28.6 \%$ & $28.6 \%$ & $0.0 \%$ & $35.7 \%$ & $21.4 \%$ & $21.4 \%$ & $7.1 \%$ & $21.4 \%$ & $42.9 \%$ \\
\hline & $\begin{array}{l}\text { Service } \\
\text { workers }\end{array}$ & $48.5 \%$ & $35.6 \%$ & $20.8 \%$ & $60.4 \%$ & $35.6 \%$ & $22.8 \%$ & $31.7 \%$ & $43.6 \%$ & $77.2 \%$ \\
\hline & $\begin{array}{l}\text { Agriculture, } \\
\text { forestry, } \\
\text { fisheries } \\
\text { workers }\end{array}$ & $60.9 \%$ & $44.9 \%$ & $35.5 \%$ & $70.3 \%$ & $28.3 \%$ & $17.4 \%$ & $21.7 \%$ & $37.0 \%$ & $84.1 \%$ \\
\hline & $\begin{array}{l}\text { Craft and } \\
\text { trade workers }\end{array}$ & $60.3 \%$ & $36.8 \%$ & $30.9 \%$ & $72.1 \%$ & $41.2 \%$ & $20.6 \%$ & $35.3 \%$ & $50.0 \%$ & $83.8 \%$ \\
\hline & $\begin{array}{l}\text { Plant and } \\
\text { machine } \\
\text { operators }\end{array}$ & $51.0 \%$ & $31.4 \%$ & $19.6 \%$ & $54.9 \%$ & $39.2 \%$ & $25.5 \%$ & $25.5 \%$ & $43.1 \%$ & $74.5 \%$ \\
\hline & $\begin{array}{l}\text { Elementary } \\
\text { occupations }\end{array}$ & $61.8 \%$ & $34.5 \%$ & $32.7 \%$ & $67.3 \%$ & $30.9 \%$ & $16.4 \%$ & $25.5 \%$ & $43.6 \%$ & $83.6 \%$ \\
\hline & Military & $50.0 \%$ & $50.0 \%$ & $50.0 \%$ & $100 \%$ & $50.0 \%$ & $0.0 \%$ & $0.0 \%$ & $50.0 \%$ & $100 \%$ \\
\hline
\end{tabular}




\begin{tabular}{|c|c|c|c|c|c|c|c|c|c|c|}
\hline \multirow[t]{2}{*}{ Category } & \multirow[t]{2}{*}{ Criteria } & \multicolumn{4}{|c|}{ Small cetacean products } & \multicolumn{4}{|c|}{ Humpback whale products } & \multirow{2}{*}{$\begin{array}{l}\text { Any } \\
\text { cetacean- } \\
\text { based } \\
\text { food } \\
\text { product }\end{array}$} \\
\hline & & Meat & Blubber & Oil & Any & Meat & Blubber & Oil & Any & \\
\hline & Students & $42.9 \%$ & $0.0 \%$ & $0.0 \%$ & $42.9 \%$ & $28.6 \%$ & $0.0 \%$ & $0.0 \%$ & $28.6 \%$ & $57.1 \%$ \\
\hline & Retired & $73.1 \%$ & $46.2 \%$ & $42.3 \%$ & $75.0 \%$ & $26.9 \%$ & $15.4 \%$ & $15.4 \%$ & $30.8 \%$ & $76.9 \%$ \\
\hline & ND & $65.5 \%$ & $38.3 \%$ & $31.1 \%$ & $68.6 \%$ & $19.7 \%$ & $10.6 \%$ & $15.2 \%$ & $24.6 \%$ & $76.5 \%$ \\
\hline \multirow{14}{*}{$\begin{array}{l}\text { Home } \\
\text { region }\end{array}$} & Barrouallie & $89.5 \%$ & $52.6 \%$ & $42.1 \%$ & $92.1 \%$ & $10.5 \%$ & $2.6 \%$ & $5.3 \%$ & $13.2 \%$ & $92.1 \%$ \\
\hline & Bequia & $42.9 \%$ & $14.8 \%$ & $5.4 \%$ & $42.9 \%$ & $73.9 \%$ & $53.7 \%$ & $61.1 \%$ & $81.8 \%$ & $83.7 \%$ \\
\hline & $\begin{array}{l}\text { Greater } \\
\text { Kingstown }\end{array}$ & $72.9 \%$ & $41.7 \%$ & $37.5 \%$ & $75.0 \%$ & $27.1 \%$ & $12.5 \%$ & $16.7 \%$ & $33.3 \%$ & $79.2 \%$ \\
\hline & Mayreau & $60.7 \%$ & $46.4 \%$ & $28.6 \%$ & $71.4 \%$ & $35.7 \%$ & $28.6 \%$ & $35.7 \%$ & $42.9 \%$ & $78.6 \%$ \\
\hline & $\begin{array}{l}\text { Mesopotamia } \\
\text { Valley }\end{array}$ & $68.4 \%$ & $42.1 \%$ & $26.3 \%$ & $68.4 \%$ & $21.1 \%$ & $0.0 \%$ & $0.0 \%$ & $21.1 \%$ & $68.4 \%$ \\
\hline & ND & $56.3 \%$ & $31.3 \%$ & $12.5 \%$ & $62.5 \%$ & $50.0 \%$ & $37.5 \%$ & $37.5 \%$ & $56.3 \%$ & $75.0 \%$ \\
\hline & non-SVG & $37.5 \%$ & $25.0 \%$ & $12.5 \%$ & $37.5 \%$ & $12.5 \%$ & $18.8 \%$ & $18.8 \%$ & $25.0 \%$ & $56.3 \%$ \\
\hline & $\begin{array}{l}\text { North } \\
\text { Leeward }\end{array}$ & $65.1 \%$ & $38.8 \%$ & $37.2 \%$ & $73.6 \%$ & $5.4 \%$ & $0.8 \%$ & $1.6 \%$ & $7.0 \%$ & $75.2 \%$ \\
\hline & $\begin{array}{l}\text { North } \\
\text { Windward }\end{array}$ & $54.9 \%$ & $33.3 \%$ & $41.2 \%$ & $62.7 \%$ & $15.7 \%$ & $0.0 \%$ & $3.9 \%$ & $19.6 \%$ & $64.7 \%$ \\
\hline & South & $80.0 \%$ & $60.0 \%$ & $45.7 \%$ & $85.7 \%$ & $25.7 \%$ & $0.0 \%$ & $2.9 \%$ & $25.7 \%$ & $88.6 \%$ \\
\hline & $\begin{array}{l}\text { South } \\
\text { Leeward }\end{array}$ & $70.5 \%$ & $56.8 \%$ & $47.0 \%$ & $79.5 \%$ & $12.9 \%$ & $3.8 \%$ & $7.6 \%$ & $18.2 \%$ & $81.1 \%$ \\
\hline & $\begin{array}{l}\text { South } \\
\text { Windward }\end{array}$ & $75.8 \%$ & $47.0 \%$ & $43.9 \%$ & $83.3 \%$ & $16.7 \%$ & $3.0 \%$ & $3.0 \%$ & $18.2 \%$ & $83.3 \%$ \\
\hline & Union Island & $38.8 \%$ & $37.9 \%$ & $24.3 \%$ & $55.3 \%$ & $29.1 \%$ & $21.4 \%$ & $32.0 \%$ & $44.7 \%$ & $63.1 \%$ \\
\hline & Windward & $70.3 \%$ & $37.8 \%$ & $21.6 \%$ & $73.0 \%$ & $16.2 \%$ & $0.0 \%$ & $0.0 \%$ & $16.2 \%$ & $73.0 \%$ \\
\hline
\end{tabular}


Table 4

Statistical relevance of selected* characteristics in predicting consumption of cetacean-based food products in SVG.

\begin{tabular}{|c|c|c|c|c|c|}
\hline \multirow[t]{2}{*}{ Category } & \multirow[t]{2}{*}{ Criteria } & \multicolumn{2}{|c|}{ Small cetacean products } & \multicolumn{2}{|c|}{ Humpback whale products } \\
\hline & & $\begin{array}{l}\text { Odds ratio of } \\
\text { consumption }\end{array}$ & $\begin{array}{l}95 \% \\
\text { Confidence } \\
\text { interval }\end{array}$ & $\begin{array}{l}\text { Odds ratio of } \\
\text { consumption }\end{array}$ & $\begin{array}{l}95 \% \\
\text { Confidence } \\
\text { interval }\end{array}$ \\
\hline \multirow[t]{2}{*}{ Gender } & Female (reference) & 1.000 & NA & 1.000 & NA \\
\hline & Male & 2.175 & $(1.495,3.185)$ & 2.440 & $(1.597,3.772)$ \\
\hline \multirow[t]{10}{*}{ Occupation } & Managers & 1.329 & $(0.622,2.979)$ & 1.693 & $(0.742,3.829)$ \\
\hline & Professionals & 0.693 & $(0.282,1.717)$ & 1.064 & $(0.339,3.187)$ \\
\hline & $\begin{array}{l}\text { Technicians and associate } \\
\text { professionals }\end{array}$ & 0.598 & $(0.158,2.181)$ & 0.367 & $(0.076,1.655)$ \\
\hline & Clerical support workers & 0.363 & $(0.073,1.451)$ & 0.078 & $(0.004,0.515)$ \\
\hline & Service and sales workers & 1.083 & $(0.618,1.918)$ & 0.845 & $(0.439,1.604)$ \\
\hline & $\begin{array}{l}\text { Skilled agricultural, forestry } \\
\text { and fishery workers }\end{array}$ & 0.647 & $(0.384,1.092)$ & 1.266 & $(0.713,2.238)$ \\
\hline & $\begin{array}{l}\text { Craft and related trades } \\
\text { workers }\end{array}$ & 0.913 & $(0.456,1.878)$ & 1.274 & $(0.619,2.597)$ \\
\hline & $\begin{array}{l}\text { Plant and machine operators, } \\
\text { and assemblers }\end{array}$ & 0.335 & $(0.159,0.701)$ & 0.765 & $(0.328,1.744)$ \\
\hline & Elementary occupations & 0.961 & $(0.454,2.066)$ & 1.654 & $(0.754,3.600)$ \\
\hline & Other or not stated (reference) & 1.000 & NA & 1.000 & NA \\
\hline \multirow[t]{12}{*}{$\begin{array}{l}\text { Home } \\
\text { region }\end{array}$} & Barrouallie & 7.404 & $\begin{array}{l}(2.509 \\
31.756)\end{array}$ & 0.073 & $(0.023,0.193)$ \\
\hline & Bequia & 0.471 & $(0.304,0.722)$ & 2.725 & $(1.669,4.561)$ \\
\hline & Greater Kingstown & 2.298 & $(1.048,5.482)$ & 0.265 & $(0.118,0.571)$ \\
\hline & Mayreau & 2.211 & $(0.800,6.807)$ & 0.485 & $(0.172,1.335)$ \\
\hline & Mesopotamia Valley & 1.308 & $(0.432,4.205)$ & 0.069 & $(0.010,0.280)$ \\
\hline & North Leeward & 1.916 & $(1.145,3.281)$ & 0.048 & $(0.021,0.100)$ \\
\hline & North Windward & 1.487 & $(0.744,3.098)$ & 0.156 & $(0.064,0.344)$ \\
\hline & South & 3.120 & $(1.200,9.723)$ & 0.207 & $(0.075,0.515)$ \\
\hline & South Leeward & 2.992 & $(1.779,5.227)$ & 0.122 & $(0.065,0.219)$ \\
\hline & South Windward & 3.207 & $(1.642,6.777)$ & 0.146 & $(0.068,0.292)$ \\
\hline & Union Island & 0.815 & $(0.479,1.386)$ & 0.446 & $(0.250,0.781)$ \\
\hline & Windward & 1.891 & $(0.843,4.560)$ & 0.060 & $(0.014,0.183)$ \\
\hline
\end{tabular}

\section{Demographic patterns}

Certain segments of the Vincentian population are more likely to consume cetacean-based food products than others. For example, male respondents reported higher levels of consumption than female respondents with $82.8 \%$ of male respondents reporting that they consume at least one of the six cetacean-based food products compared to $69.2 \%$ of female respondents. 
The relative rates of consumption vary slightly when analyzed by individual food product (meat, blubber, and oil; each from small cetaceans and humpback whales) but maintain the overall trend toward higher consumption rates among male respondents than among female respondents for all products. Overall, male respondents are 2.2 times more likely $(p<0.001)$ to consume food products from small cetaceans, and 2.4 times more likely $(p<0.001)$ to consume food products from humpback whales, than female respondents.

We found that the age of the respondent offered little to no influence on the consumption of cetacean-based food products. None of the seven age categories significantly differed from the rest in terms of consumption rates.

With regard to the effect of occupational category, as a proxy for socioeconomic class, we found lower consumption rates of food products derived from small cetaceans at associated only with respondents with occupations in Category 8 (Plant and machine operators) $(p=0.004)$, and for products derived from humpback whales only with respondents in Category 4 (Clerical workers) $(p=0.025)$.

Across all demographics, meat is a more popular food product than blubber or oil, whether from small cetaceans or humpback whales. Meat from small cetaceans is consumed by $59.7 \%$ of all respondents, while $30.3 \%$ of respondents reported consuming meat from humpback whales. This may be compared to the results for consumption of blubber and oil, which are $37.7 \%$ and $29.4 \%$, respectively, for small cetaceans and $17.7 \%$ and $22.0 \%$, respectively, for humpback whales.

\section{Spatial variation}

Cetacean-based food products are more popular in some parts of SVG than in others (Fig. 2). Food products derived from small cetaceans are more popular than those derived from humpback whales in all regions of the main island, as well as on the Grenadine island of Mayreau, while the opposite is true on Bequia and Union Island. Respondents who claimed hometowns outside of SVG tended to prefer small cetacean-based food products to those derived from humpback whales and those for whom we had no hometown data had mixed results. Overall, cetacean-based food products are most popular in the regions where they are produced: respondents from Barrouallie are 7.4 times more likely $(p<0.001)$ to consume food products derived from small cetaceans than respondents from other regions. Correspondingly, respondents from Bequia are 2.7 times more likely $(p<0.001)$ to consume food products derived from humpback whales than respondents from other regions.

Rates of small cetacean-based food product consumption generally decrease in regions progressively further from Barrouallie. Meat from small cetaceans is consumed by $65.1 \%$ and $70.5 \%$ of respondents in regions immediately adjacent to Barrouallie (North Leeward and South Leeward, respectively) but by $54.9 \%$ of respondents in the North Windward region, the furthest region on the main island-by road-from Barrouallie. Similarly, food products derived from humpback whales are more popular among respondents from Bequia, where they are produced, with humpback whale meat, blubber, and oil consumed by $73.9 \%, 53.7 \%$, and $61.1 \%$ of respondents in that region, respectively. Rates of humpback whale-based food product consumption are generally higher within the Grenadines than on the main island. For example, meat from humpback whales is consumed by $35.7 \%$ and $29.1 \%$ of respondents from the other two Grenadine islands surveyed-Mayreau and Union Island, respectively-as compared to rates ranging from $5.4-27.1 \%$ across the main island's nine regions with a rate of $14.2 \%$ for St. Vincent as a whole.

Other attributes of cetacean-based food product consumption follow geographically-defined patterns. For example, a respondent's preferred whaling operation-the small-cetacean operation at Barrouallie and the humpback whale operation at Bequia-can be predicted geographically. Specifically, the majority (68.3\%) of those who consume cetacean-based food products choose products that come from only one of the country's two whaling operations. This preference is stronger among consumers of small cetacean products than consumers of products derived from humpback whales. Among consumers of small cetacean products, $66.1 \%$ do not consume humpback whale products, while $35.1 \%$ of consumers of humpback whale products do not consume products from small cetaceans. 
Results indicate that, among those who do consume cetacean-based food products, the most common frequency for products derived from small cetaceans is greater than once per month but less than once per week (27.7\% of respondents who consume). For products derived from humpback whales, the most common frequency is greater than once per year but less than once per month (19.2\% of respondents who consume) (Fig. 3). A minority (5.1\%) of respondents reported a perceived seasonality regarding cetacean-based food product availability, but were not in agreement as to when the seasons of relative abundance or scarcity occurred.

The amount of small cetacean tissue, by individual species and all species combined (including and excluding killer whales), that can be consumed each week without exceeding the PTWI is shown in Table 5. Using the mean percent MeHg for all species combined, on average, blubber can be consumed in the highest quantity without exceeding the PTWI, followed by kidney, liver, and muscle. If killer whales are excluded from the calculations (as currently pending legislation would ban the take of killer whales), the tissue ranking remains the same, but consumers could eat $13.7 \%$ more blubber, $8.7 \%$ more liver, $7.1 \%$ more kidney, and $21.5 \%$ more muscle. 
Table 5

Minimum, mean, and maximum safe consumption amounts* (in grams)

\begin{tabular}{|c|c|c|c|c|c|c|c|c|c|c|c|}
\hline \multirow[b]{2}{*}{ Species } & \multirow[b]{2}{*}{ Tissue } & \multirow[b]{2}{*}{$n$} & \multicolumn{3}{|c|}{ Min \% MeHg } & \multicolumn{3}{|c|}{ Mean \% MeHg } & \multicolumn{3}{|c|}{ Max \% MeHg } \\
\hline & & & $\begin{array}{l}\% \\
\mathrm{MeHg}\end{array}$ & $\begin{array}{l}\text { Mean } \\
\text { (g) }\end{array}$ & $\begin{array}{l}\text { Range } \\
\text { (g) }\end{array}$ & $\begin{array}{l}\% \\
\mathrm{MeHg}\end{array}$ & $\begin{array}{l}\text { Mean } \\
\text { (g) }\end{array}$ & $\begin{array}{l}\text { Range } \\
\text { (g) }\end{array}$ & $\begin{array}{l}\% \\
\mathrm{MeHg}\end{array}$ & $\begin{array}{l}\text { Mean } \\
\text { (g) }\end{array}$ & $\begin{array}{l}\text { Range } \\
\text { (g) }\end{array}$ \\
\hline \multirow[t]{4}{*}{$\begin{array}{l}\text { Combined (all } \\
\text { species) }\end{array}$} & Muscle & 59 & 12.7 & 255 & $\begin{array}{l}3.7- \\
1105\end{array}$ & 62.2 & 52.1 & $\begin{array}{l}0.76- \\
226\end{array}$ & 100 & 32.4 & $\begin{array}{l}0.48- \\
140\end{array}$ \\
\hline & Blubber & 36 & 4.2 & 2218 & $\begin{array}{l}300- \\
18253\end{array}$ & 7.0 & 1331 & $\begin{array}{l}180- \\
10952\end{array}$ & 9.7 & 960 & $\begin{array}{l}130- \\
7903\end{array}$ \\
\hline & Liver & 17 & 0.48 & 1673 & $\begin{array}{l}18.4- \\
4992\end{array}$ & 7.7 & 104 & $\begin{array}{l}1.1- \\
311\end{array}$ & 24.2 & 33.2 & $\begin{array}{l}0.36- \\
99.0\end{array}$ \\
\hline & Kidney & 10 & 3.4 & 1970 & $\begin{array}{l}4.3- \\
9630\end{array}$ & 18.4 & 364 & $\begin{array}{l}0.79- \\
1780\end{array}$ & 22.7 & 295 & $\begin{array}{l}0.64- \\
1442\end{array}$ \\
\hline \multirow{4}{*}{$\begin{array}{l}\text { Combined } \\
\text { (excluding killer } \\
\text { whale) }\end{array}$} & Muscle & 47 & 12.7 & 309 & $\begin{array}{l}4.1- \\
1105\end{array}$ & 61.9 & 63.3 & $\begin{array}{l}0.83- \\
227\end{array}$ & 100 & 39.2 & $\begin{array}{l}0.52- \\
140\end{array}$ \\
\hline & Blubber & 30 & 4.2 & 2522 & $\begin{array}{l}388- \\
18253\end{array}$ & 7.0 & 1513 & $\begin{array}{l}233- \\
10952\end{array}$ & 9.7 & 1092 & $\begin{array}{l}168- \\
7903\end{array}$ \\
\hline & Liver & 12 & 0.48 & 1938 & $\begin{array}{l}18.4- \\
4213\end{array}$ & 8.2 & 113 & $\begin{array}{l}1.1- \\
247\end{array}$ & 24.2 & 38.4 & $\begin{array}{l}0.36- \\
83.6\end{array}$ \\
\hline & Kidney & 8 & 7.6 & 1098 & $\begin{array}{l}11.0- \\
4308\end{array}$ & 21.4 & 390 & $\begin{array}{l}3.9- \\
1530\end{array}$ & 22.7 & 368 & $\begin{array}{l}3.7- \\
1442\end{array}$ \\
\hline \multirow[t]{4}{*}{ Killer whale } & Muscle & 12 & ND & ND & ND & 71.0 & 8.0 & $\begin{array}{l}0.67- \\
20.8\end{array}$ & ND & ND & ND \\
\hline & Blubber & 6 & ND & ND & ND & $7.0 * \star$ & 420 & $\begin{array}{l}180- \\
1218\end{array}$ & ND & ND & ND \\
\hline & Liver & 5 & ND & ND & ND & 1.9 & 262 & $\begin{array}{l}5.2- \\
1261\end{array}$ & ND & ND & ND \\
\hline & Kidney & 2 & ND & ND & ND & 3.4 & 29.1 & $\begin{array}{l}4.3- \\
53.9\end{array}$ & ND & ND & ND \\
\hline \multirow[t]{4}{*}{ False killer whale } & Muscle & 21 & ND & ND & ND & 36.0 & 142 & $\begin{array}{l}10.3- \\
390\end{array}$ & ND & ND & ND \\
\hline & Blubber & 5 & ND & ND & ND & $7.0 * \star$ & 625 & $\begin{array}{l}233- \\
876\end{array}$ & ND & ND & ND \\
\hline & Liver & 2 & ND & ND & ND & $7.7 * \star$ & 4.1 & $\begin{array}{l}1.1- \\
7.1\end{array}$ & ND & ND & ND \\
\hline & Kidney & 2 & ND & ND & ND & $18.4^{\star \star}$ & 49.1 & $\begin{array}{l}4.6- \\
93.5\end{array}$ & ND & ND & ND \\
\hline \multirow[t]{4}{*}{$\begin{array}{l}\text { Short-finned pilot } \\
\text { whale }\end{array}$} & Muscle & 21 & 12.7 & 219 & $\begin{array}{l}4.1- \\
834\end{array}$ & 49.9 & 55.7 & $\begin{array}{l}1.0- \\
212\end{array}$ & 81.0 & 34.3 & $\begin{array}{l}0.64- \\
131\end{array}$ \\
\hline & Blubber & 23 & ND & ND & ND & 5.4 & 2075 & $\begin{array}{l}548- \\
14197\end{array}$ & ND & ND & ND \\
\hline & Liver & 9 & 0.48 & 2353 & $\begin{array}{l}108- \\
4213\end{array}$ & 5.1 & 221 & $\begin{array}{l}10.2- \\
396\end{array}$ & 9.8 & 115 & $\begin{array}{l}5.3- \\
206\end{array}$ \\
\hline & Kidney & 5 & ND & ND & ND & 14.6 & 889 & $\begin{array}{l}46.8- \\
2243\end{array}$ & ND & ND & ND \\
\hline
\end{tabular}




\begin{tabular}{|c|c|c|c|c|c|c|c|c|c|c|c|}
\hline \multirow[b]{2}{*}{ Risso's dolphin } & \multirow[b]{2}{*}{ Muscle } & \multirow[b]{2}{*}{3} & \multicolumn{3}{|c|}{ Min \% MeHg } & \multicolumn{3}{|c|}{ Mean \% MeHg } & \multicolumn{3}{|c|}{ Max \% MeHg } \\
\hline & & & 44.0 & 88.1 & $\begin{array}{l}7.7- \\
194\end{array}$ & 60.8 & 63.7 & $\begin{array}{l}5.6- \\
141\end{array}$ & 75.9 & 51.1 & $\begin{array}{l}4.4- \\
113\end{array}$ \\
\hline & Blubber & 1 & ND & ND & ND & $7.0^{\star * *}$ & 5083 & ND & ND & ND & ND \\
\hline & Liver & 0 & 1.5 & ND & ND & 5.9 & ND & ND & 10.3 & ND & ND \\
\hline & Kidney & 0 & 10.1 & ND & ND & 16.4 & ND & ND & 22.7 & ND & ND \\
\hline \multirow[t]{4}{*}{ Stenella spp. } & Muscle & 2 & 35.0 & 97.1 & $\begin{array}{l}63.1- \\
131\end{array}$ & 69.9 & 48.6 & $\begin{array}{l}31.6- \\
65.6\end{array}$ & 100 & 34.0 & $\begin{array}{l}22.1- \\
45.9\end{array}$ \\
\hline & Blubber & 1 & 4.2 & 603 & ND & 7.5 & 337 & ND & 9.7 & 261 & ND \\
\hline & Liver & 1 & 1.6 & 586 & ND & 9.7 & 96.6 & ND & 24.2 & 38.7 & ND \\
\hline & Kidney & 1 & 7.6 & 13.3 & ND & 24.8 & 4.1 & ND & 45.0 & 2.2 & ND \\
\hline
\end{tabular}

Reasons for not consuming

We received a wide range of descriptive responses to our questions about reasons why cetacean-based food products were not consumed by those who reported not consuming them and categorized these responses into thirteen broad rejection categories (Table 6). The most common reason for rejection of cetacean-based food products was simple personal preference (38.7\% of rejections of small cetacean-based food products and $35.1 \%$ rejections of humpback whale-based food products). Respondents whose rejections fell into this category remarked that the appearance, smell, taste, or texture of the food products was unappealing. Following preference, availability and religion are the next most common reasons for the rejection of cetacean-based food products from both small cetaceans ( $21.6 \%$ for both categories) and humpback whales ( $25.1 \%$ and $19.9 \%$, respectively). Certain religious traditions (primarily the Rastafarian and Seventh-Day Adventist faiths) forbid the consumption of cetacean-based food products and the products are not available to the same degree in all parts of the country. Following these top three, rejections due to perceptions of the unhealthiness of the food products $(6.8 \%$ of small cetacean rejections and $4.7 \%$ of humpback whale rejections) were the next most common. We understand the perception of "unhealthiness" to be based upon other characteristics of the food products other than Hg contamination, as this issue was not widely known at the time of our fieldwork-indeed, it remains so today. Other reasons for rejection included abstention because cetaceans are mammals (3.1\% of small cetacean rejections and $3.7 \%$ of humpback whale rejections), the belief that cetaceans menstruate (3.1\% of small cetacean rejections and $2.1 \%$ of humpback whale rejections), perceptions about the unsanitary nature of the relevant food-production methods $(2.1 \%$ of small cetacean rejections but not mentioned for humpback whales), the ethics of whaling (3.1\% of humpback whale rejections but not mentioned for small cetaceans), and the food products not fitting within the respondent's diet ( $2.1 \%$ of humpback whale rejections but less than $1.0 \%$ for small cetaceans). Less common reasons for rejection, all given by less than $1.0 \%$ of those who rejected a particular cetacean-based food product, included the prohibitive cost of the food products, the decision not to eat fish (coupled with the belief that cetaceans are fish), and the belief that whaling is illegal. 
Table 6

Reasons for not consuming cetacean-based food products, measured across the entire study area.

\begin{tabular}{|c|c|c|c|}
\hline $\begin{array}{l}\text { Rejection } \\
\text { reason } \\
\text { category }\end{array}$ & Explanation & $\begin{array}{l}\text { Percent of small } \\
\text { cetacean product } \\
\text { rejections }\end{array}$ & $\begin{array}{l}\text { Percent of humpback } \\
\text { whale product } \\
\text { rejections }\end{array}$ \\
\hline Availability & $\begin{array}{l}\text { Considers cetacean-based food products to be } \\
\text { unavailable in respondent's hometown }\end{array}$ & $21.6 \%$ & $25.1 \%$ \\
\hline Cost & $\begin{array}{l}\text { Considers cetacean-based food products to be too } \\
\text { expensive }\end{array}$ & $0.7 \%$ & - \\
\hline Diet & $\begin{array}{l}\text { Cetacean-based food products are not part of } \\
\text { respondent's chosen diet (vegetarian, vegan, etc.) }\end{array}$ & $0.7 \%$ & $2.1 \%$ \\
\hline Ethics & Considers whaling to be unethical & - & $3.1 \%$ \\
\hline Fish & $\begin{array}{l}\text { Does not consume fish, and considers cetaceans to } \\
\text { be fish }\end{array}$ & $0.7 \%$ & - \\
\hline Health & $\begin{array}{l}\text { Considers cetacean-based food products to be } \\
\text { unhealthy }\end{array}$ & $6.8 \%$ & $4.7 \%$ \\
\hline Legality & Believes whaling to be illegal & - & $1.0 \%$ \\
\hline Mammal & Does not consume meat from mammals & $3.1 \%$ & $3.7 \%$ \\
\hline Menstruation & $\begin{array}{l}\text { Believes cetaceans menstruate and does not } \\
\text { consume them because of this (see discussion } \\
\text { below) }\end{array}$ & $3.1 \%$ & $2.1 \%$ \\
\hline Other & Uncategorized & $1.0 \%$ & $3.1 \%$ \\
\hline Preference & $\begin{array}{l}\text { Does not like the appearance, smell, taste, or texture } \\
\text { of cetacean-based food products }\end{array}$ & $38.7 \%$ & $35.1 \%$ \\
\hline Religion & $\begin{array}{l}\text { Considers the consumption of cetacean-based food } \\
\text { products to violate respondent's religion }\end{array}$ & $21.6 \%$ & $19.9 \%$ \\
\hline Sanitation & $\begin{array}{l}\text { Considers cetacean-based food products to be } \\
\text { produced, sold, or stored in unsanitary conditions }\end{array}$ & $2.1 \%$ & - \\
\hline
\end{tabular}

The spatial variation to reasons for rejection is illustrated in Fig. 4. Availability is most commonly given in regions geographically distant from the regions of origin for the respective food products: Barrouallie for small cetaceans and Bequia for humpback whales. For example, the highest rates of small cetacean rejection for the reason of availability are found in the Grenadines (41.1\% on Bequia, $40.0 \%$ on Mayreau, and $15.9 \%$ on Union Island), along with the North Windward region of St. Vincent, where $35.0 \%$ of those who gave a reason for rejection cited lack of availability. Availability also drives the rejection of food products derived from humpback whales: Bequia saw the lowest incidence of this reason at just $4.5 \%$. Pockets of frequency appear for other rejection reasons, such as the high prevalence of religious rejections in Bequia (11.1\% for small cetaceans and $27.7 \%$ for humpback whales), the Mesopotamia Valley (83.3\% for small cetaceans and $40.0 \%$ for humpback whales) and the South Windward region (41.2\% for small cetaceans and $40.0 \%$ for humpback whales). 
Table 7

Forty-two-year comparison of wet-weight THg concentrations in muscle tissue of Eastern Caribbean small cetaceans.

\begin{tabular}{|llll|}
\hline Year & $\begin{array}{l}\text { Average THg ww concentration in short-finned pilot } \\
\text { whales (Globicephala macrorhynchus) }\end{array}$ & $\begin{array}{l}\text { Average THg ww concentration } \\
\text { in spinner dolphins }\end{array}$ & Source \\
\hline 1978 & $3.99 \mu \mathrm{g} / \mathrm{g}(\mathrm{n}=5)$ & $1.1 \mu \mathrm{g} / \mathrm{g}(\mathrm{n}=2)$ & $\begin{array}{l}\text { Gaskin et al. } \\
1974\end{array}$ \\
\hline 2014 & - & $1.57 \mu \mathrm{g} / \mathrm{g}(\mathrm{n}=28)$ & $\begin{array}{l}\text { Fielding and } \\
\text { Evans } 2014\end{array}$ \\
\hline 2020 & $20.5 \mu \mathrm{g} / \mathrm{g}(\mathrm{n}=21)$ & $3.22 \mu \mathrm{g} / \mathrm{g}(\mathrm{n}=2) \star$ & McCormack et \\
& & & al. 2020 \\
\hline
\end{tabular}

\section{Discussion}

While cetacean-based food products are highly popular among the Vincentian public, distinct demographic and geographic patterns define this popularity, indicating that risks presented by the contaminants in these food products are unevenly distributed within the population.

\section{Demographic patterns}

This gender-based preference for consumption, coupled with the fact that $39 \%$ of households in SVG are headed by women (Ministry of Finance 2019) and that women, regardless of whether they are considered the heads of their households, are the primary planners of household meals (Young 1993), indicates that dietary guidelines, if established, may be effective in protecting the population from pollution-associated negative health effects.

The fact that age is not a significant demographic variable in patterns of cetacean-based food product consumption challenges the common conception that whaling-and, by extension, the consumption of food products derived from whaling -is a declining way of life globally and specifically in the Caribbean. A 2013 study found that $64 \%$ and $55 \%$ of respondents ( $n=211$, ages 16-25, mean 18.0 years) consumed meat and blubber, respectively, from small cetaceans (Fielding 2013). Our findings indicate that cetacean-based food products remain popular with the Vincentian youth and that their popularity neither increases nor decreases significantly with the age of the consumer.

Our analysis of consumption data organized by occupational categories (as a proxy for socioeconomic class) suggests findings that differ from those of previous studies. For example, Adams $(1980,25)$ found that small cetacean meat was occasionally consumed by what he termed "low income informants," and hardly at all by those of higher socioeconomic standing. By contrast, we found no significant preference for cetacean-based food products among those of lower socioeconomic classes (occupational categories 6-9), nor by respondents who reported being unemployed at the time of the survey. While our findings did show some significance in predicting consumption among respondents representing occupational categories 4 and 8 (Clerical workers and Plant and machine operators), the prediction in both of these cases was toward lower than average rates of consumption. These socioeconomic findings may indicate either that ours is a more thorough assessment of dietary patterns than previous studies, likely owing to our larger sample size and partnership with local collaborators, or that dietary patterns have changed during the intervening decades.

\section{Spatial variation}

The two regions in which Vincentian whaling operations are based-Barrouallie and Bequia-are home to the highest rates of consumption of the products derived from those operations. Each of these regions also represent a nadir of consumption of the "other" cetacean-based food product (small cetacean products in Bequia and humpback whale products in Barrouallie). This spatial pattern could be understood as simply a matter of availability: local demand is sufficiently high and transportation logistics are complicated or expensive to the point that it often makes sense for vendors to distribute food 
products close to their point of origin. Indeed, with a few exceptions, our results suggest that consumption rates strongly correlate with proximity to the whaling centers of Barrouallie and Bequia. A general association of small cetacean-based food products with the main island of St. Vincent and humpback whale-based food products with the Grenadines confirms this trend. Within the Grenadines, only the tiny island of Mayreau (pop. 270, $n=28$ ) hosts a population whose demand for small cetacean-based food products matches that of any region on the main island. No region on St. Vincent exceeds any Grenadine island in terms of humpback whale meat, blubber, or oil consumption but the region in which these products are most popular is the South-the closest region, geographically, to the Grenadines. Simple availability does not fully account for the low rate of small cetacean product consumption in Bequia and the similarly low rate for humpback whale products in Barrouallie. Rather, the pattern explained by proximity to whaling centers is likely punctuated by specific regional pride and loyalty to local products within each of the whaling centers.

\section{Frequency and amount of consumption}

The frequency and amount of consumption are major considerations that help fine-tune our understanding of $\mathrm{Hg}$ exposure beyond what a binary analysis of consumption versus abstention allows. One who consumes cetacean-based food products, but does so very infrequently or in small amounts, is not as exposed to $\mathrm{Hg}$ as one who consumes frequently or who consumes very large portions.

In other whaling communities where health authorities have advised the public on reducing their exposure to environmental contaminants, dietary recommendations typically have been designed to limit the frequency of consumption. For example, the first dietary recommendations in the Faroe Islands (a North Atlantic whaling community), issued in 1977, suggested the general population limit its consumption of small cetacean meat and blubber to no more than one meal per week. Over the following decades, as concentrations of $\mathrm{Hg}$ and other contaminants continued to increase, Faroese health authorities issued increasingly strict dietary guidelines, culminating in the controversial 2008 recommendation that "the pilot whale... no longer [be] used for human consumption" (Weihe and Joensen 2012, 18594). During the 31 years that elapsed between the first dietary advice and the 2008 recommendation to discontinue consumption, health authorities were able to inculcate the Faroese public to the nature of the risk and the associated need for mitigation (Fielding 2018). Owing to the higher levels of $\mathrm{Hg}$ found in cetaceans taken in SVG, relative to those in the Faroe Islands, the Vincentian population is unlikely to have the luxury of such a long process of acclimatization.

Among nutrition researchers, portion size is a difficult datum to gather accurately (Cypel et al. 1997). With regard to small cetacean meat and blubber, specifically, our analysis might have been aided by the fact that these food products are typically sold in single-serving bundles. A comparison of small cetacean meat bundles, however, found that they varied by more than $100 \mathrm{~g}$, ranging from $113 \mathrm{~g}$ to $227 \mathrm{~g}$, even though prices were equal among portions of all sizes (Fielding 2010). This makes our respondents' statements on portion size such as "five dollars' worth every week," or "a bundle a day," less useful in terms of actual portion size analysis. Most of our respondents provided detailed information on the frequency with which they consumed cetacean-based food products but could not specify their typical portion size in a meaningful way. In the absence of reliable data on portion sizes, we will assume a bundle of dried meat weighing $170 \mathrm{~g}$-the midpoint of the range presented by Fielding (2010) -as an estimate for average portion size until future research provides more specific data.

The amounts that can be safely consumed, based upon the PTWI (JECFA 2006), can be instructive in terms of dietary advice and public health advocacy. Given that the species identity of the cetacean being consumed is only reliably known for products resulting from the operation targeting humpback whales in Bequia (Fielding 2014), we shall address here only two measures given in Table 5: the mean amount that can be consumed based upon the mean MeHg percentage from the literature for all small cetacean species combined and the same value for all species combined, excluding killer whales. In the current system, with all species combined, a $60 \mathrm{~kg}$ adult can consume slightly less than a third (31\%) of an averageweight bundle $(170 \mathrm{~g})$ of small cetacean meat per week and remain within the guidelines provided by the FAO/WHO. If killer whales were removed from the analysis, that typical adult could consume slightly more than a third (37\%) of an average bundle per week. 
Large intra- and interspecies variability exists in the amount of tissue that could be consumed without exceeding the PTWI. This variability is likely a result of the large variability in $\mathrm{THg}$ concentrations and the percent present as MeHg, which is further confounded by the fact McCormack et al. (2020) did not speciate for MeHg and we, therefore, had to rely on values taken from the literature. As a result, we cannot make specific recommendations as to the safest species to consume, particularly for muscle, but suggest that the most conservative interpretation would be to focus upon the minimum amount of tissue calculated based upon the highest percentage $\mathrm{MeHg}$ (the low end of the range presented in each row of the rightmost column in Table 5). While the greatest amount of blubber could be consumed without exceeding the PTWI, consumers should still be cautious because this estimation is only based on $\mathrm{MeHg}$ and does not include lipophilic organic contaminants such as PCBs and pesticides, which are often found in cetacean blubber (Dam and Bloch 2000). Finally, it is also important to consider that consumers may eat different tissues, or tissues from various species, within a week and these values are based on the consumption of a single tissue.

\section{Reasons for not consuming}

An analysis of the reasons for not consuming cetacean-based food products illuminates the existence of certain barriers that, if removed, would likely result in greater rates of consumption throughout the study area. Of particular interest is the spatiality of rejection responses due to lack of availability. The lowest frequency of availability-related rejections of each food product is, predictably, in the location of its production: Barrouallie for small cetaceans and Bequia for humpback whales. One can infer the reach of the formal and informal distribution routes through the maps presented in Fig. 2 and Fig. 4: food products derived from small cetaceans are conveyed well to all regions of St. Vincent except the north windward coast, while its distribution in the Grenadines appears not to meet demand. Similarly, humpback whale products are welldistributed in Bequia but less so in the Southern Grenadines. Humpback whale product distribution in St. Vincent appears to be patchy-most likely a result of the informal nature of the distribution networks there.

Lack of availability limits the consumption of humpback whale products, especially in regions of St. Vincent, and the same is a major reason for the lack of small cetacean product consumption in the Grenadines, as well as in the North Windward region of St. Vincent. Going by road, North Windward is the region of the island that is the most difficult and time-consuming to reach from Barrouallie; it seems that the travel is not worth the reward to many of the island's small cetacean product vendors. The necessity of a long and expensive ferry trip ( $>6$ hours to cover the $65 \mathrm{~km}$ from St. Vincent to Union Island, at EC\$100 [US\$37] for a round-trip fare, based upon the available ferry options in 2018) certainly contributes to the reduced availability of small cetacean products in the Grenadines as well.

Some respondents perceive a "seasonality" regarding cetacean-based food product availability. While the Bequia whaling operation does, in fact, rely upon the seasonal migrations of humpback whales between the Eastern Caribbean and North Atlantic (Martin et al. 1984; Stevick et al. 1999, 2018), the Barrouallie-based small cetacean operation shows no clear seasonal variation except for the short-term breaks in whaling activity during certain holidays and periods of hazardous weather (Adams 1973; Fielding 2018). The perceived seasonality in whaling among consumers may, rather, indicate seasonal variation to the distribution networks that bring cetacean-based food products throughout the country.

Among consumers of small cetacean products, $62.8 \%$ do not consume humpback whale products, while only $31.9 \%$ of consumers of humpback whale products do not consume products from small cetaceans. This is likely an indication not only of the ubiquity of products derived from small cetaceans but also of their tendency to replace humpback whale meat when the latter is unavailable. At the time of our study, the Bequia operation had successfully landed only one humpback whale during the previous three years $(2015,2016$, and 2017). Rejections for lack of availability indicate that unmet demand for cetacean-based food products likely exists in some parts of SVG. Specifically, if more whaling were to occur, and if distribution networks were expanded to reach currently underserved areas, the products would likely find ready consumers. The Bequia humpback whale operation is limited to a maximum of four whales per year by the IWC. The Barrouallie small cetacean operation, however, is not limited by Vincentian domestic law or by any international treaty to which SVG is party (Fielding 2018). In theory, the operation acts as an open-access resource institution and-if cetacean populations can sustain 
the increased pressure-could potentially expand to fulfill demand for cetacean-based food products in places where availability is currently limited. This underscores the pressing need for future research to understand the size and dynamics of local cetacean populations. It also illustrates the need for future policy action, specifically the establishment of whaling quotas or another system of limited entry to regulate the take of small cetaceans by Vincentian whalers.

Rejection of cetacean-based food products for religious reasons is largely associated with two specific religious groups: adherents to the Rastafarian and Seventh-Day Adventist faiths. Both traditions proscribe the consumption of sea creatures lacking "fins and scales" in keeping with their interpretation of the Levitical dietary laws given in the Bible. In the 2012 census, $11.6 \%$ of Vincentians self-identified as Seventh-Day Adventists, while 1.1\% identified themselves as Rastafarians. Rejections by Adventists dominate the religious rejection of cetacean-based food products. Of all the rejections for religious reasons, $71.4 \%$ of small cetacean product rejections and $73.7 \%$ of humpback whale product rejections are by respondents who cited their Adventist faith; $11.1 \%$ and $7.9 \%$ are from those who cited their Rastafarian faith, and $17.5 \%$ and $18.4 \%$ are for unspecified religious adherence.

We found more religious rejections of small cetacean products than of humpback whale products along with the occurrence-absent in the small cetacean product rejections-of ethics and legality as a category of reasons not to consume humpback whale meat. The Bequia-based whaling operation has attracted more international scrutiny than the small cetacean operation in Barrouallie. This is, in part, due to the fact that humpback whales are internationally protected and are strictly managed by the IWC. Under current IWC rules, every six years the government of SVG must submit a "need statement" to the IWC, laying out the cultural and nutritional benefit of maintaining its aboriginal subsistence whaling quota (IWC 2012, 2015). The operation targeting small cetaceans falls only under the jurisdiction of the Government of SVG because small cetaceans in the Caribbean region are not managed internationally (Gillespie 2001; Reeves 2005). The differential management of the two whaling operations may give Vincentian consumers the impression that Bequia's operation targeting humpback whales is more ethically and legally ambiguous than the Barrouallie operation, since the former requires international oversight and the latter does not.

Although rejection for "menstruation" is mentioned only infrequently in our surveys (3.1\% and $2.1 \%$ for small cetacean and humpback whale products, respectively), it warrants discussion here for its unusual nature. Specifically, a belief exists among some members of the Vincentian public that female cetaceans menstruate and that this constitutes a valid reason to eschew their consumption. To be clear, cetaceans do not menstruate, although certain odontocetes, including killer whales and short-finned pilot whales, do experience a reduction and eventual loss of fertility with age (Marsh and Kasuya 1984, 1986) - a phenomenon that some scholars have compared to menopause in humans (McAuliffe and Whitehead 2005). While the origins of this unfounded belief remain unknown, it calls to mind the general menstrual taboos long described by anthropologists (e.g. Kamsler 1938; Stephens 1961; Young and Bacdayan 1965; Montgomery 1974) and in particular, the interactions between the menstrual taboo and hunting traditions (Kitahara 1982). The important difference in the cases analyzed by Kitahara and the Vincentian case is that the former considers only human menstruation as a taboo during hunting, while the latter projects this characteristic upon the animal.

Previous research has found that cetacean-based food products are largely viewed as healthy in SVG. A 2013 study found that $68 \%$ of survey respondents $(n=211)$ believed small cetacean meat to be healthy (Fielding 2013). A further $19 \%$ of respondents in that study answered that they did not know whether it was healthy or not. Only $8 \%$ responded that small cetacean meat was not healthy. In our surveys, a minority of respondents-6.8\% and $4.7 \%$-cite health as their reason for not consuming small cetacean meat and humpback whale meat, respectively. Even when all of the marginally health-related categories of reasons for rejection (diet, fish, health, mammal, and sanitation) are taken together, only $13.4 \%$ and $10.5 \%$ of respondents reject small cetacean products and humpback whale products, respectively, for health. Considering the findings of McCormack et al. (2020), it is clear that this public perception is misaligned with the reality of environmental contamination.

\section{Conclusions}

Page 20/29 
Our findings indicate a high rate of consumption of cetacean-based food products, at a time when contaminant concentrations have been shown to have increased during recent decades. The only extant studies of $\mathrm{Hg}$ pollution in cetaceans taken for human consumption in the Caribbean are described in a paper by Gaskin et al. (1974), the pilot project to the McCormack study (Fielding and Evans 2014), and the McCormack et al. (2020) study itself. These results are shown for comparison in Table 7. The nearly three-fold increase in THg concentrations for spinner dolphins and the five-fold increase for short-finned pilot whales during the 42 years covered by these studies is likely due to both the refinement of analytical methods and an actual increase in contaminant release into the marine environment (Pirrone et al. 2010).

[Table 7 here]

McCormack et al. (2020) advised that their study warranted further investigation, owing to the potential human health issue uncovered therein. We would go further, recommending that, given the high concentration of environmental contaminants and the high degree of popularity of these food products, the Government of SVG should embark upon a demographically and geographically targeted intervention campaign by establishing and communicating dietary recommendations based upon the most recent information available. Our analysis of the demographic and geographic patterns of the consumption of cetacean-based food products will allow an information campaign to be directed toward those members of the population with the greatest risk of exposure. Given the variation in $\mathrm{Hg}$ concentrations reported by species, we would also recommend that species-specific whaling quotas be considered as a way to eliminate the most contaminated food products from the local Vincentian food system.

\section{Abbreviations}

AIC - Akaike information criteria

EC\$ - Eastern Caribbean dollar

FAO - Food and Agricultural Organisation of the United Nations

$\mathrm{Hg}$ - mercury

IWC - International Whaling Commission

MeHg - methylmercury

PTWI - provisional tolerable weekly intake

SVG - Saint Vincent \& the Grenadines

$\mathrm{THg}$ - total mercury

WHO - World Health Organization

\section{Declarations}

\section{Ethics approval and consent to participate}

The human-subject research methods and acquisition of verbal consent for this study were approved by the Institutional Review Board at the University of the South under permit number 18-13.

\section{Consent for publication}

Not applicable 
The datasets produced and analyzed for this study are available from the corresponding author (Fielding) upon reasonable request.

\section{Competing interests}

The authors declare that they have no competing interests.

\section{Funding}

Funding for this research was provided by the Ross University School of Veterinary Medicine, the University of the South and the Institute of Environment (Florida International University). Specific funding units at the latter institution include the Department of Earth \& Environmental Systems, the Faculty Research Grants Fund, the McCrickard Faculty Development Fund, the Office of Civic Engagement, and the Office of Undergraduate Research. The funding bodies had no influence upon the design of the study and collection, analysis, and interpretation of data and in writing the manuscript.

\section{Authors' contributions}

RF, CM, and VR designed and led the study. MAM and JD determined safe weekly consumption amounts. ADO and ME conducted the spatial analysis and designed the cartography. JA led the statistical analysis with assistance from NAD and SS. HMG and HT wrote early drafts of the religious and demographic sections, respectively. RF, JJK, CM, MAM, JD, and JA were major contributors in writing the manuscript. All authors read and approved the final manuscript.

\section{Acknowledgements}

The authors gratefully acknowledge the support of Don Bergfelt and Fortune Sithole from Ross University School of Veterinary Medicine. We appreciate the assistance of student field researchers from the University of the South who are not listed as coauthors: Elissa Clark, Helena Kilburn, Isabel Kirby, Komal Kunwar, Lauren Newman, and Connor Peach. This research would not have been possible without the partnership of the following local field collaborators: Alana Adams, Moriah Alves, Enrico Barker, Sheena Clouden, Samantha Ince, April Jacobs, Keaah Johnson, Otmar Marshall, Shakera Ollivierre, Aureil Phillips, Tiffany Phillips, Tishana Reid, Anthony Sargeant, Eugena Simmons, and Simone Williams. Data collection was conducted with the approval of-but independently from-the appropriate Ministries of the Government of St. Vincent \& the Grenadines: The Ministry of Health, Wellness \& the Environment and the Ministry of Agriculture, Forestry, Fisheries, Rural Transformation, Industry \& Labour.

\section{Reference}

1. Adams JE. Fish preferences and prejudices in a small Caribbean island: A study of fish consumption patterns in St. Vincent based on a household survey. Proc Annu Gulf Caribb Fish Inst. 1980;32:15-34.

2. Adams JE. Shore whaling in St. Vincent, West Indies. Caribb Q. 1973;19(4):42-50.

3. Adams JE. Historical Geography of Whaling in Bequia Island, West Indies. Caribb Q. 1971;11(3):55-74.

4. Alter SE, Simmonds MP, Brandon IR. Forecasting the consequences of climate-driven shifts in human behavior on cetaceans. Mar Policy. 2010;34:943-54.

5. Arima S, Nagakura K. Mercury and selenium content of odontoceti. Bull Jpn Soc Sci Fish. 1979;45:623-6.

6. Bustamante P, Garrigue C, Breau L, Caurant F, Dabin W, Greaves J, Dodemont R. Trace elements in two odontocete species (Kogia breviceps and Globicephala macrorhynchus) stranded in New Caledonia (South Pacific). Environ Pollut. 2003;124:263-71. https://doi.org/10.1016/S0269-7491(02)00480-3.

7. Caldwell DK, Caldwell MC. Dolphin and small whale fisheries of the Caribbean and West Indies: occurrence, history, and catch statistics-with special reference to the Lesser Antillean Island of St. Vincent. Can J Fish Aquat Sci. 
1975;32(7):1105-10.

8. Capelli R, Das K, De Pellegrini R, Drava G, Lepoint G, Miglio C, Minganti V, Poggi R. Distribution of trace elements in organs of six species of cetaceans from the Ligurian Sea (Mediterranean), and the relationship with stable carbon and nitrogen ratios. Sci Total Environ. 2008;390:569-78. https://doi.org/10.1016/j.scitotenv.2007.10.036.

9. Cardellicchio N, Decataldo A, Di Leo A, Misino A. Accumulation and tissue distribution of mercury and selenium in striped dolphins (Stenella coeruleoalba) from the Mediterranean Sea (southern Italy). Environ Pollut. 2002;116:265-71. https://doi.org/10.1016/S0269-7491(01)00127-0.

10. $10.1016 /$ S0025-326X(02)00095-4

Chen MH, Shih CC, Chou CL, Chou LS. 2002. Mercury, organic-mercury and selenium in small cetaceans in Taiwanese waters. Mar Pollut Bull 45: 237-245 (2002). https://doi.org/10.1016/S0025-326X(02)00095-4.

11. Clapham P, Van Waerebeek K. Bushmeat and bycatch: the sum of the parts. Molec Ecol. 2007;16(13):2607-9.

12. Coleman JS. Relational analysis: The study of social organizations with survey methods. Hum Organ 17: 28-36 (195859).

13. Cypel YS, Guenther PM, Petot GJ. Validity of portion-size measurement aids: A review. J Am Diet Assoc. 1997;97(3):289-92.

14. Dam M, Bloch D. Screening of mercury and persistent organochlorine pollutants in long-finned pilot whale (Globicephala melas) in the Faroe Islands. Mar Pollut Bull. 2000;40(12):1090-9. https://doi.org/10.1016/S0025-326X(00)00060-6.

15. Endo T, Haraguchi K. High mercury levels in hair samples from residents of Taiji, a Japanese whaling town. Mar Pollut Bull. 2010;60(5):743-7.

16. Endo T, Haraguchi K, Hotta Y, Hisamichi Y, Lavery S, Dalebout ML, Baker CS. Total mercury, methyl mercury, and selenium levels in the red meat of small cetaceans sold for human consumption in Japan. Environ Sci Technol. 2005;39:5703-8. https://doi.org/10.1021/es050215e.

17. Endo T, Kimura O, Hisamichi Y, Minoshima Y, Haraguchi K, Kakumoto C, Kobayashi M. Distribution of total mercury, methyl mercury, and selenium in pod of killer whales (Orcinus orca) stranded in the northern area of Japan: comparison of mature females with calves. Environ Pollut. 2006;144:145-50. https://doi.org/10.1016/j.envpol.2005.12.035.

18. FAO/WHO. Report of the Joint FAO/WHO Expert Consultation on the Risks and Benefits of Fish Consumption. Rome: Food and Agriculture Organization of the United Nations/Geneva: World Health Organization; 2011.

19. Fielding R. The Wake of the Whale: Hunter Societies in the Caribbean and North Atlantic. Cambridge: Harvard University Press; 2018.

20. Fielding R. The liminal coastline in the life of a whale: Transition, identity, and food-production in the Eastern Caribbean. Geoforum. 2014;54:10-6.

21. Fielding R. Whaling futures: A survey of Faroese and Vincentian youth on the topic of artisanal whaling. Soc Nat Resour. 2013;26(7):810-26.

22. Fielding R. Artisanal Whaling in the Atlantic: a Comparative Study of Culture, Conflict, and Conservation in St. Vincent and the Faroe Islands. Ph.D. thesis, Louisiana State University, 2010.

23. Fielding R, Ollivierre AD. Saint Vincent and the Grenadines. In: Allen C, editor. Landscapes and Landforms of the Lesser Antilles. World Geomorphological Landscapes 12. New York: Springer; 2017. pp. 223-41.

24. Fielding R, Evans DW. Mercury in Caribbean dolphins (Stenella longirostris and Stenella frontalis) caught for human consumption off St. Vincent, West Indies. Mar Pollut Bull. 2014;89(1-2):30-4. https://doi.org/10.1016/j.marpolbul.2014.10.040.

25. Gaskin DE, Smith GJD, Arnold PW, Louisy MV, Frank R, Holdrinet M, McWade JW. Mercury. DDT, dieldrin, and PCB in two species of Odontoceti (Cetacea) from St. Lucia, Lesser Antilles. J Fish Res Board Can. 1974;31:1235-9.

https://doi.org/10.1139/f74-147.

Page 23/29 
26. Gillespie A. Small cetaceans, international law and the International Whaling Commission. Melb J Int Law. 2001;2(2):257-303.

27. Grandjean P. Only One Chance: How Environmental Pollution Impairs Brain Development-and How to Protect the Brains of the Next Generation. Oxford: Oxford University Press; 2013.

28. ILO [International Labour Office]. International Standard Classification of Occupations ISCO-08. Geneva: International Labour Office; 2012.

29. Itano K, Kawai S, Miyazaki N, Tatsukawa R, Fujiyama T. Mercury and selenium levels in striped dolphins caught off the Pacific coast of Japan. Agric Biol Chem. 1984;48(5):1109-16.

30. IWC [International Whaling Commission]. Report of the IWC Expert Workshop on Aboriginal Subsistence Whaling (ASW), IWC/66/ASW Rep01 (2015).

31. IWC [International Whaling Commission]. Annex H. Summary Need Statement on Behalf of St Vincent and The Grenadines, IWC/64/ASW Rep03 (2012).

32. James RJ, Lafeuillee MX, Li G, Salinas, Savchenko Y. Explaining High Unemployment in ECCU Countries. IMF Working Paper WP/19/144. (2019).

33. JECFA (Joint FAO/WHO Expert Committee on Food Additives). Evaluation of certain food additives and contaminants. 67th report, Rome, Italy, 2006.

34. JMHLW (Japanese Ministry of Health, Labor, and Welfare). Advice for pregnant women on fish consumption concerning mercury contamination. Joint subcommittees on animal origin foods and toxicology under the food sanitation committee and the pharmaceutical affairs and food sanitation council. Japanese Ministry of Health, Labor and Welfare, 2003.

35. Josupeit H. Consumption patterns for fish and seafood in the Caribbean with special emphasis on bivalves and univalves. In A. Lovatelli and S. Sarkis, editors. A regional shellfish hatchery for the Wider Caribbean: Assessing its feasibility and sustainability. FAO Regional Technical Workshop. 18-21 October 2010, Kingston, Jamaica. FAO Fisheries and Aquaculture Proceedings 19. Rome: FAO, 2011. 199-223.

36. Kamsler H. Hebrew Menstrual Taboos. J Am Folk. 1938;51(199):76-82.

37. Kehrig HA, Costa M, Moreira I, Malm O. Total and methylmercury in a Brazilian estuary, Rio de Janeiro. Mar Pollut Bull. 2002;44(10):1018-23.

38. Kitahara M. Menstrual taboos and the importance of hunting. Am Anthropol. 1982;84(4):901-3.

39. Kojadinovic J, Potier M, Le Corre M, Cosson RP, Bustamante P. Mercury content in commercial pelagic fish and its risk assessment in the Western Indian Ocean. Science Total Environ. 2006;366(2-3):688-700.

40. Marsh H, Kasuya T. Changes in the ovaries of the short-finned pilot whale, Globicephala macrorhynchus, with age and reproductive activity. Reports of the International Whaling Commission. 1984;6:311-35.

41. Marsh H, Kasuya T. Evidence for reproductive senescence in female cetaceans. Reports of the International Whaling Commission. 1986;8:57-74.

42. Martin AR, Katona SK, Matilla D, Hembree D, Waters TD. Migration of Humpback Whales between the Caribbean and Iceland. J Mamm. 1984;65(2):330-3.

43. McAuliffe $\mathrm{K}$, Whitehead $\mathrm{H}$. Eusociality, menopause and information in matrilineal whales. Trends Ecol Evol. 2005;20(12):650.

44. McCormack MA, Fielding R, Kiszka JJ, Paz V, Jackson BP, Bergfelt DR, Dutton J. Mercury and selenium concentrations, and selenium:mercury molar ratios, in small cetaceans taken off St. Vincent, West Indies. Environ Res. 2020;181:108908.

45. Ministry of Finance. Planning E. Sustainable Development \& Information Technology, St. Vincent and the Grenadines. Population and Demography, 2019. http://www.stats.gov.vc/.

46. Montgomery RE. A cross-cultural study of menstruation, menstrual taboos, and related social variables. Ethos. 1974;2:137-70.

Page 24/ 29 
47. Olmedo P, Hernández AF, Pla A, Femia P, Navas-Acien A, Gil F. Determination of essential elements (copper, manganese, selenium and zinc) in fish and shellfish samples. Risk and nutritional assessment and mercury-selenium balance. Food Chem Toxicol. 2013;62:299-307.

48. Palmisano F, Cardellicchio N, Zambonin PG. Speciation of mercury in dolphin liver: a two-stage mechanism for the demethylation accumulation process and role of selenium. Mar Environ Res. 1995;40(2):109-21.

49. Pirrone N, Cinnirella S, Feng X, Finkelman RB, Friedli HR, Leaner J, Mason R, Mukherjee AB, Stracher GB, Streets DG, Telmer K. Global mercury emissions to the atmosphere from anthropogenic and natural sources. Atmos Chem Phys. 2010;10:5951-64.

50. R Core Team. R: A language and environment for statistical computing. R Foundation for Statistical Computing, Vienna, Austria, 2016. https://www.R-project.org/.

51. Reeves RR. Insights on Marine Mammals of the Wider Caribbean Sea Region (including the Gulf of Mexico). Derived from Whaling Documents. Paper presented at the Regional Workshop of Experts on the Development of the Marine Mammal Action Plan for the Wider Caribbean Region. Bridgetown, Barbados. July 18-21, 2005.

52. Robards MD, Reeves RR. The global extent and character of marine mammal consumption by humans: 1970-2009. Biol Conserv. 2011;144(12):2770-86.

53. Romero A, Cresswell J. In the land of the mermaid: How culture, not ecology, influenced marine mammal exploitation in the Southeastern Caribbean. In: Romero A, West SE, editors. Environmental Issues in Latin America and the Caribbean. New York: Springer; 2005. pp. 3-30.

54. Ruelas-Inzunza JR, Horvat M, Pérez-Cortés H, Páez-Osuna F. Methylmercury and total mercury distribution in tissues of gray whales (Eschrichtius robustus) and spinner dolphins (Stenella longirostris) stranded along the lower Gulf of California, Mexico. Cienc Mar. 2003;29(1):1-8.

55. Sakamoto M, Itai T, Yasutake A, Iwasaki T, Yasunaga G, Fujise Y, Nakamura M, Murata K, Chan HM, Domingo JL, Marumoto M. Mercury speciation and selenium in toothed-whale muscles. Environ Res. 2015;143:55-61.

56. Simmonds MP, Haraguchi K, Endo T, Cipriano F, Palumbi SR, Troisi GM. Human health significance of organochlorine and mercury contaminants in Japanese whale meat. J Toxicol Environ Health Part A. 2002;65(17):1211-35.

57. Stephens WN. A cross-cultural study of menstrual taboos. Genet Psychol Monogr. 1961;64:385-416.

58. Stevick PT, Bouveret L, Gandilhon N, Rinaldi C, Rinaldi R, Broms F, Carlson C, Kennedy A, Ward N, Wenzel F. Migratory destinations and timing of humpback whales in the southeastern Caribbean differ from those off the Dominican Republic. J Cetac Res Manage. 2018;18:127-33.

59. Stevick PT, Carlson CA, Balcomb KC. A note on the migratory destinations of humpback whales from the eastern Caribbean. J Cetac Res Manage. 1999;1(3):251-4.

60. Storelli MM, Ceci E, Marcotrigiano GO. Comparison of total mercury, methylmercury, and selenium in muscle tissues and in the liver of Stenella coeruleoalba (Meyen) and Caretta caretta (Linnaeus). Bull Environ Contam Toxicol. 1998;61:5417.

61. Storelli MM, Zizzo N, Marcotrigiano GO. Heavy metals and methylmercury in tissues of Risso's dolphin (Grampus griseus) and Cuvier's beaked whale (Ziphius cavirostris) stranded in Italy (South Adriatic Sea). Bull Environ Contam Toxicol. 1999;63:703-10.

62. Wagemann R, Trebacz E, Hunt R, Boila G. Percent methylmercury and organic mercury in tissues of marine mammals and fish using different experimental and calculation methods. Environ Toxicol Chem. 1997;16(9):1859-66.

63. Ward N, Blows. Mon, Blows! A History of Bequia Whaling. Woods Hole: Gecko Productions; 1995.

64. Weihe $P$, Joensen HD. Dietary recommendations regarding pilot whale meat and blubber in the Faroe Islands. Int $J$ Circumpolar Health. 2012;71(1):18594. http://dx.doi.org/10.3402/ijch.v71i0.18594.

65. Wise JP Jr, Wise JTF, Wise CF, Wise SS, Gianios C Jr, Xie H, Walter R, Boswell M, Zhu C, Zheng T, Perkins C. A three-year study of metal levels in skin biopsies of whales in the Gulf of Mexico after the Deepwater Horizon oil crisis. Comp

Page 25/29 
Biochem Phys C. 2018;205:15-25.

66. Wise JP Jr, Wise JT, Wise CF, Wise SS, Zhu C, Browning CL, Zheng T, Perkins C, Gianios C Jr, Xie H, Wise Sr JP. Metal levels in whales from the Gulf of Maine: A one environmental health approach. Chemosphere. 2019a;216:653-60.

67. Wise JP Jr, Croom-Perez TJ, Meaza I, Aboueissa AM, Montalvo CAL, Martin-Bras M, Speer RM, Bonilla-Garzón A, Urbán J, Perkins C, Wise Sr JP. A whale of a tale: A one environmental health approach to study metal pollution in the Sea of Cortez. Toxicol Appl Pharm. 2019b;376:58-69.

68. Young FW, Bacdayan AA. Menstrual taboos and social rigidity. Ethnology. 1965;4(2):225-40.

69. Young VH. Becoming West Indian: Culture, Self, and Nation in St. Vincent. Washington, D.C.: Smithsonian Institution Press; 1993.

70. Zane WW. Journeys to the Spiritual Lands: The Natural History of a West Indian Religion. New York: Oxford University Press; 1999.

\section{Figures}

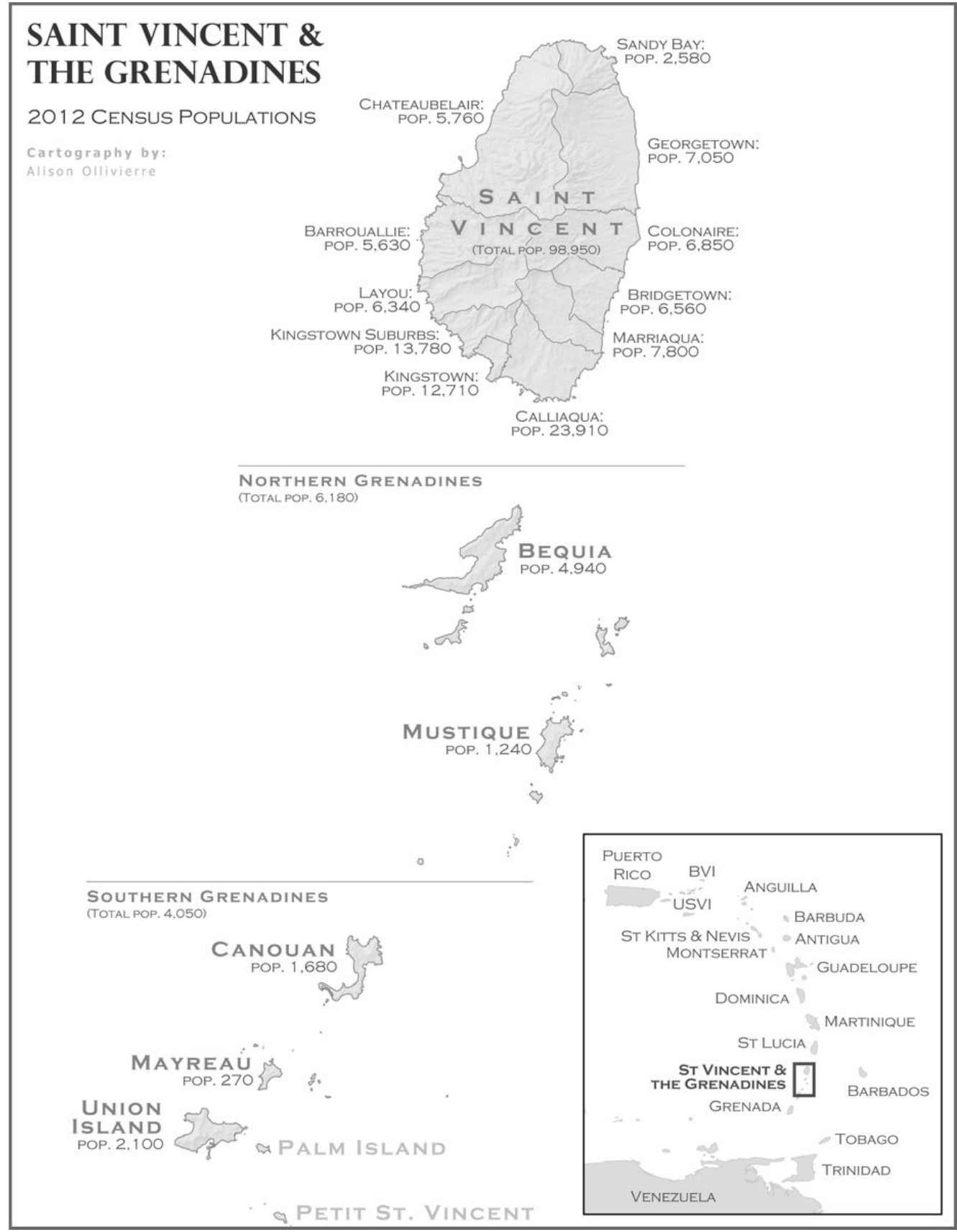

Page 26/29 
Figure 1

The study site, with 2012 population by census region (Ministry of Finance, 2019)

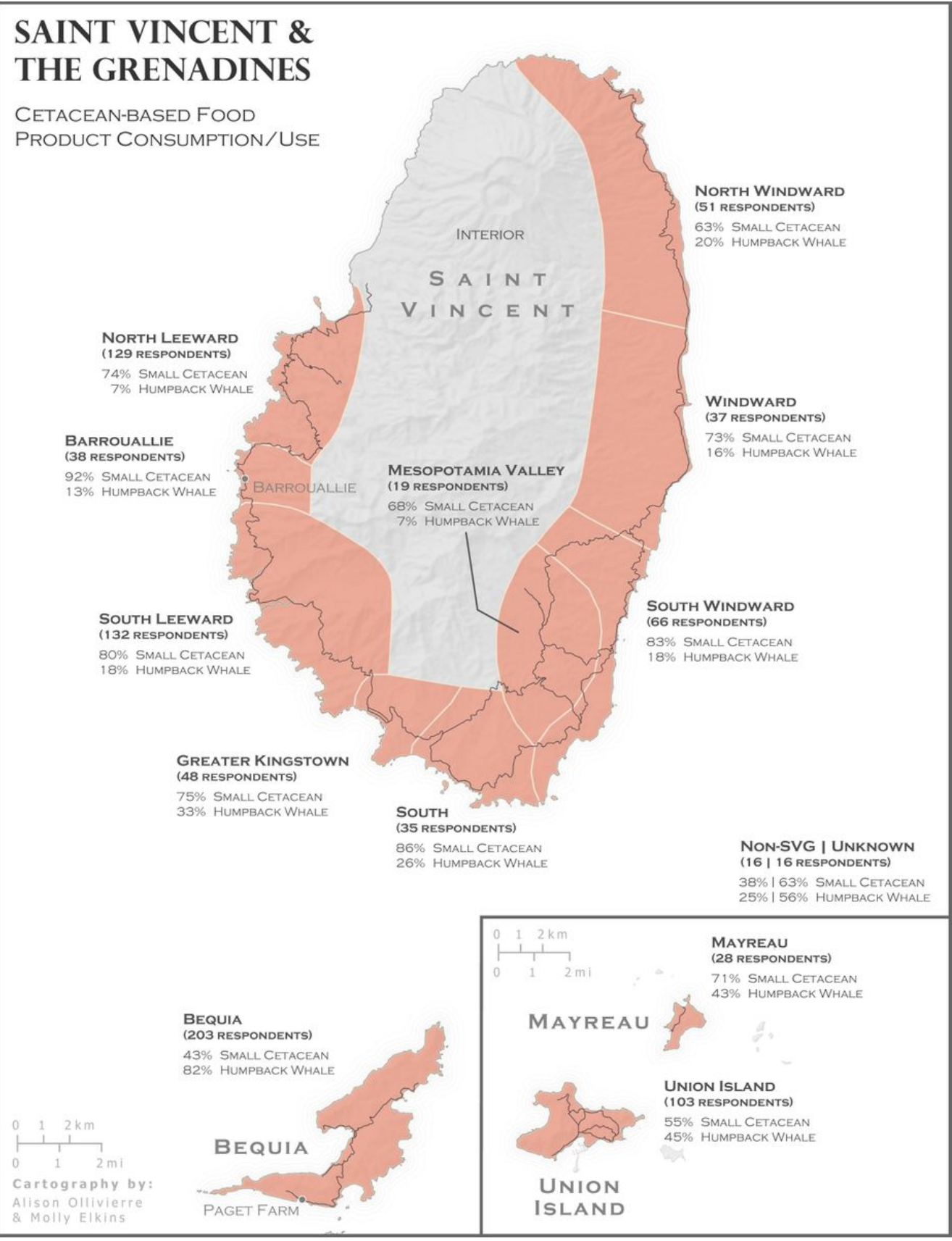

Figure 2

Geographical patterns of consumption of cetacean-based food products in SVG 


\section{CONSU M P ION FRE Q UENCY}

\section{Small Cetacean}

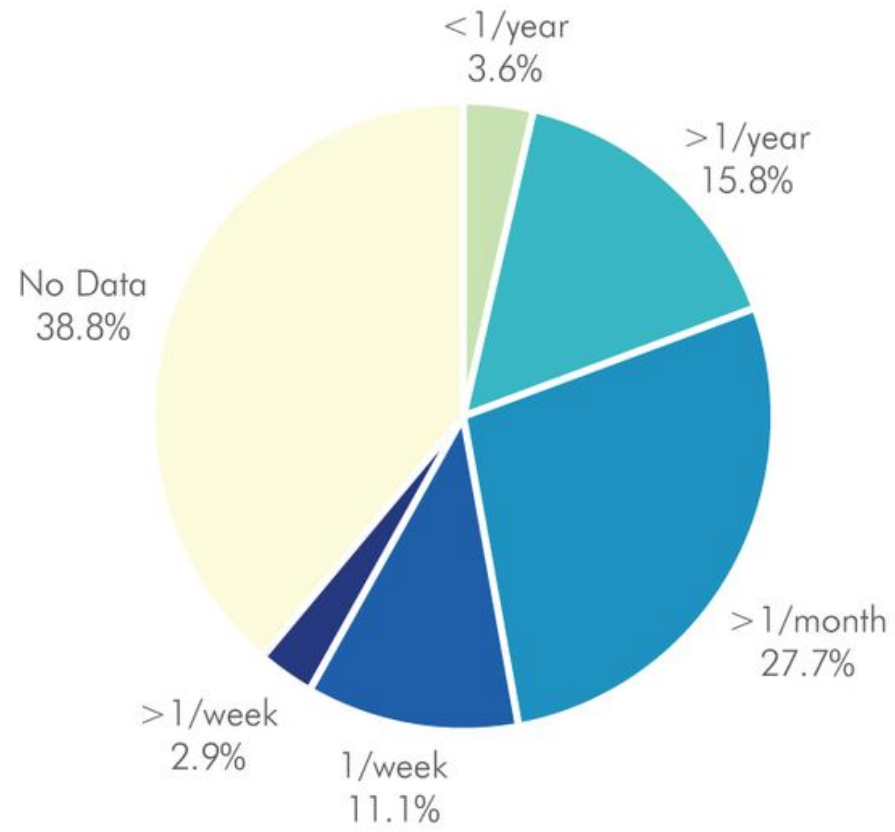

Humpback Whale

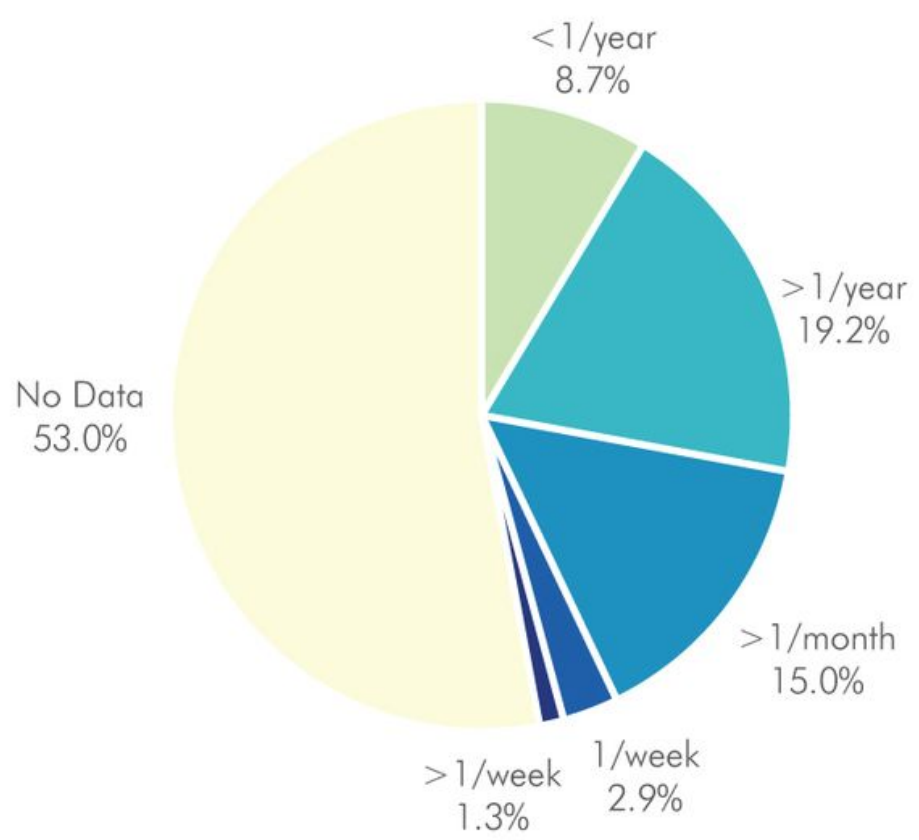

\section{Figure 3}

Frequency of consumption of cetacean-based food products in SVG 


\section{SAINT VINCENT \& \\ THE GRENADINES}

Cetacean-Based FoOd

PRODUCT REJECTION

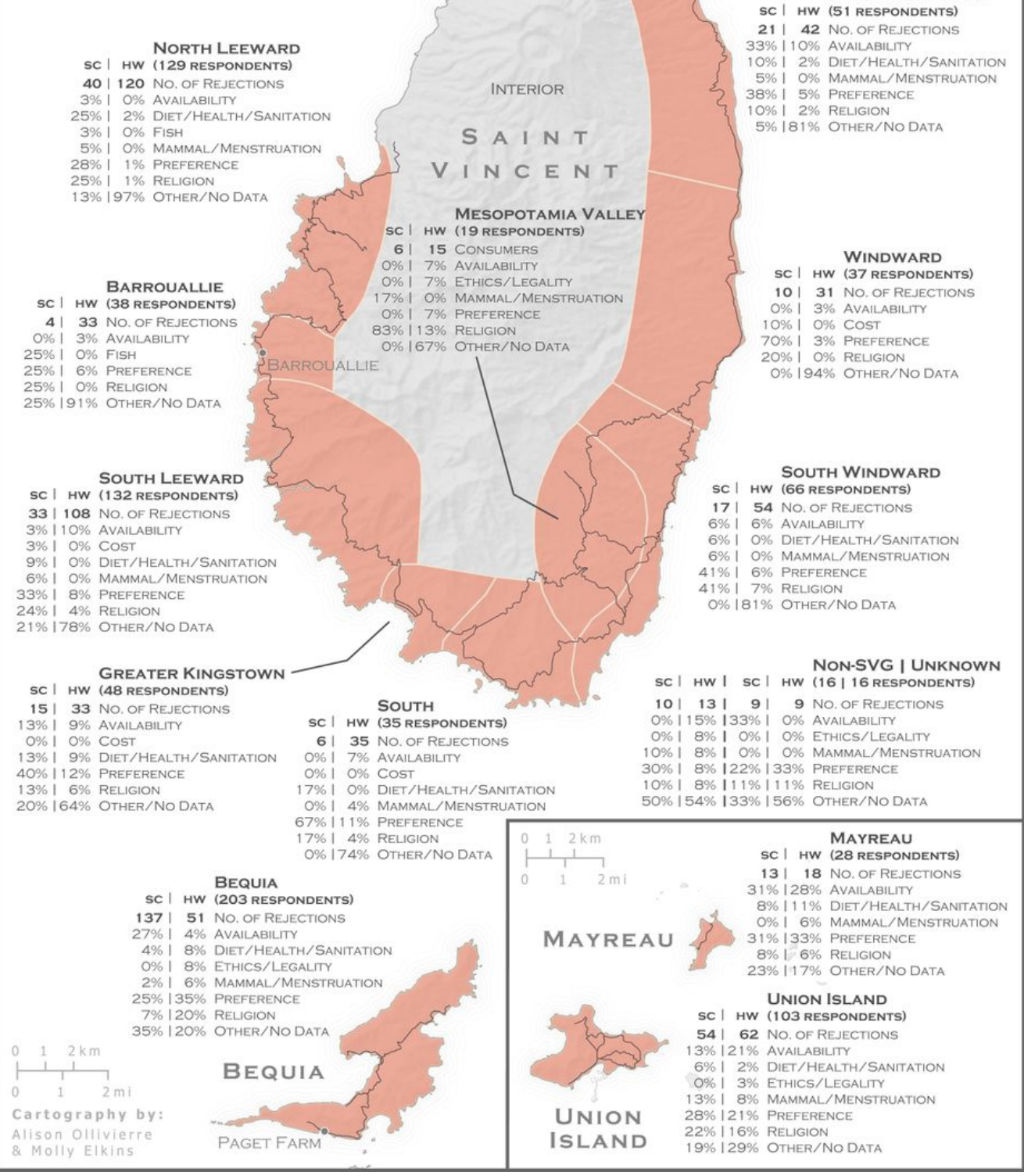

Figure 4

Geographical patterns of reasons given for not consuming cetacean-based food products in SVG 